\title{
GRAVITATIONAL AND ELECTROWEAK UNIFICATION BY REPLACING DIFFEOMORPHISMS WITH LARGER GROUP
}

\author{
DAVE PANDRES, JR.*
}

\begin{abstract}
The covariance group for general relativity, the diffeomorphisms, is replaced by a group of coordinate transformations which contains the diffeomorphisms as a proper subgroup. The larger group is defined by the assumption that all observers will agree whether any given quantity is conserved. Alternatively, and equivalently, it is defined by the assumption that all observers will agree that the general relativistic wave equation describes the propagation of light. Thus, the group replacement is analogous to the replacement of the Lorentz group by the diffeomorphisms that led Einstein from special relativity to general relativity, and is also consistent with the assumption of constant light velocity that led him to special relativity. The enlarged covariance group leads to a noncommutative geometry based not on a manifold, but on a nonlocal space in which paths, rather than points, are the most primitive invariant entities. This yields a theory which unifies the gravitational and electroweak interactions. The theory contains no adjustable parameters, such as those that are chosen arbitrarily in the standard model.
\end{abstract}

\section{INTRODUCTION}

During the past several decades, we have published nine papers in which we have pursued the suggestion by Einstein that a unified field theory be constructed by replacing the covariance group for general relativity with a larger group, analogous to the step which led from special relativity to general relativity. Our results unify the gravitational and electroweak interactions.

The purpose of this article is to survey our work in a way that makes it as easy to understand as possible. Thus, we recall only what is essential and make some changes in notation. In order to optimize readability, we give the detailed calculations justifying certain equations in an Appendix.

Key words and phrases. Gravitation, Electroweak, Unification, Enlarged Covariance Group.

*North Georgia College and State University, Dahlonega, GA 30597

Emeritus Professor of Mathematics

Current Address: 1244 Arbor Rd., \#522, Winston Salem, NC 27104

Current E-Mail Address: dpandres@triad.rr.com. 
1.1. Suggestions of Dirac and Einstein. Our research has been motivated philosophically, not only by Einstein's [1] suggestion, but also by a previous and more general suggestion by Dirac [2].

Dirac: In the Preface to the First Edition of his famous book Quantum Mechanics, Dirac made the prophetic statement: "The growth of the use of transformation theory, as applied first to relativity and later to the quantum theory, is the essence of the new method in theoretical physics. Further progress lies in the direction of making our equations invariant under wider and still wider transformations."

Einstein: In his Autobiographical Notes, Einstein made the following statement which is consistent with that of Dirac, but which is more specific: "A theory can be tested by experience, but there is no way from experience to the setting up of a theory. Equations of such complexity as are the equations of the gravitational field can be found only through the discovery of a logically simple mathematical condition which determines the equations completely or [at least] almost completely. Once one has those sufficiently strong formal conditions, one requires only a little knowledge of facts for the setting up of a theory; in the case of the equations of gravitation it is the four-dimensionality and the symmetric tensor as expression for the structure of space which, together with the invariance concerning the continuous transformation-group, determine the equations almost completely."

"Our problem is that of finding the field equations for the total field. The desired structure must be a generalization of the symmetric tensor. The group must not be any narrower than that of the continuous transformations of co-ordinates. If one introduces a richer structure, then the group will no longer determine the equations as strongly as in the case of the symmetric tensor as structure. Therefore it would be most beautiful, if one were to succeed in expanding the group once more, analogous to the step which led from special relativity to general relativity."

1.2. The Diffeomorphism Group. In the modern literature, the group that Einstein called the "continuous transformations of co-ordinates" is called the "group of diffeomorphisms." For a diffeomorphism between space-time coordinates $x^{\alpha}$ and $x^{\widetilde{\alpha}}$, infinitesimal changes in the coordinates are related by $\mathrm{d} x^{\widetilde{\alpha}}=x^{\widetilde{\alpha}}{ }_{\mu} \mathrm{d} x^{\mu}$, where repeated indices are summed over the values $0,1,2,3$. The matrix of the transformation coefficients $x^{\widetilde{\alpha}}{ }_{\mu}$ must be non-singular, and the integrability condition

$$
x^{\widetilde{\alpha}}{ }_{\mu, \nu}-x^{\widetilde{\alpha}}{ }_{\nu, \mu}=0
$$

must be satisfied. 
Ordinary partial differentiation is denoted by a comma or, when more convenient, by the symbol $\partial$. Thus, the partial derivative of $\Phi$ with respect to $x^{\mu}$ may be written $\Phi,,_{\mu}$ or $\partial_{\mu} \Phi$.

1.3. Generalization of Einstein's "Elevator" Argument. Initially [3], we merely suggested that the condition expressed by Eq. (1) be abandoned, but did not propose a condition which should replace it. We now recall our motivation for this suggestion. It is based on an argument which is a generalization of the "elevator" argument that led Einstein from special relativity to general relativity.

We begin with the special relativistic equation of motion for a free particle

$$
\frac{\mathrm{d}^{2} x^{i}}{\mathrm{~d} s^{2}}=0
$$

where $-\mathrm{d} s^{2}=g_{i j} \mathrm{~d} x^{i} \mathrm{~d} x^{j}$, and $g_{i j}=\operatorname{diag}(-1,1,1,1)$. We consider the imageequation of Eq. (2) under a transformation $\mathrm{d} x^{i}=h_{\mu}^{i} \mathrm{~d} x^{\mu}$ between coordinate increments $\mathrm{d} x^{i}$ and coordinate increments $\mathrm{d} x^{\mu}$, in which $h_{\mu, \nu}^{i}-h_{\nu, \mu}^{i} \neq 0$. Thus, Eq. (1), the integrability condition for diffeomorphisms, is not satisfied. The transformation coefficients $h_{\mu}^{i}$ are an orthonormal tetrad of vectors in terms of which the general relativistic space-time metric is expressed, i.e., $g_{\mu \nu}=g_{i j} h_{\mu}^{i} h_{\nu}^{j}$.

We find (Appendix) that the image-equation is

$$
\frac{\mathrm{d}^{2} x^{\alpha}}{\mathrm{d} s^{2}}+\Gamma_{\mu \nu}^{\alpha} \frac{\mathrm{d} x^{\mu}}{\mathrm{d} s} \frac{\mathrm{d} x^{\nu}}{\mathrm{d} s}=-f_{\nu}^{\alpha} \frac{\mathrm{d} x^{\nu}}{\mathrm{d} s}
$$

where $\Gamma_{\mu \nu}^{\alpha}$ is the usual Christoffel symbol, and $f_{\mu \nu}=v_{i} f_{\mu \nu}^{i}$, where $f_{\mu \nu}^{i}=$ $h_{\nu, \mu}^{i}-h_{\mu, \nu}^{i}$ and $v^{i}=\frac{\mathrm{d} x^{i}}{\mathrm{~d} s}$ is the (constant) first integral of $\frac{\mathrm{d}^{2} x^{i}}{\mathrm{~d} s^{2}}=0$. Thus, it appears at first glance that Eq. (3) describes a charged particle moving in a gravitational and electromagnetic field. However, $f_{\mu \nu}$ cannot be interpreted as the electromagnetic field, because the relation $v^{i}=\frac{\mathrm{d} x^{i}}{\mathrm{~d} s}=h_{\mu}^{i} \frac{\mathrm{d} x^{\mu}}{\mathrm{d} s}$ implies that $v^{i}$ depends upon $\frac{\mathrm{d} x^{\mu}}{\mathrm{d} s}$. Although $f_{\mu \nu}$ is a linear combination of the $f_{\mu \nu}^{i}$ with coefficients $v_{i}$ which are constant along the world-line of a particle, it is unsatisfactory that the values of these coefficients should depend upon $\frac{\mathrm{d} x^{\mu}}{\mathrm{d} s}$. This would imply that the electromagnetic field experienced by a particle depends upon the velocity of the particle, in disagreement with experiment. In two later papers [4,5], we tried to get a satisfactory description of gravitation and electromagnetism alone by investigating a class of theories in 
which the four antisymmetric tensors $f_{\mu \nu}^{i}$ are constant multiples of one another. However, theories in this class suffer from the defect that they are not based on a covariance group, and thus lack a "guiding principle."

The inescapable fact is that for transformations with $h_{\mu, \nu}^{i}-h_{\nu, \mu}^{i} \neq 0$, the right side of Eq. (3) contains four linearly independent antisymmetric tensors, while only one is needed to describe the electromagnetic field.

However, the analysis of this Section does suggest that Eq. (11) be abandoned, and that $f_{\mu \nu}^{0}$ might somehow describe the electromagnetic field, while $f_{\mu \nu}^{i}$ for $i \neq 0$ might somehow describe the short-range interactions.

\subsection{Consequences of Abandoning the Integrability Condition for}

Diffeomorphisms: Path-Dependence. If the integrability condition of Eq. (11) is not satisfied, it still is possible to "integrate" the relation $\mathrm{d} x^{\widetilde{\alpha}}=$ $x^{\widetilde{\alpha}}{ }_{\mu} \mathrm{d} x^{\mu}$ from the origin $o$ of an $X$ coordinate system to a terminal point $x$ with coordinates $x^{\alpha}$, and to "integrate" the inverse relation $\mathrm{d} x^{\alpha}=x_{\widetilde{\mu}}^{\alpha} \mathrm{d} x^{\widetilde{\mu}}$ from the origin $\widetilde{o}$ of an $\widetilde{X}$ coordinate system to a terminal point $\widetilde{x}$ with coordinates $x^{\widetilde{\alpha}}$. (The origins may be regarded as coordinates of observers $\Omega$ and $\widetilde{\Omega}$ who use the $X$ and $\widetilde{X}$ coordinate systems, respectively.) This gives the results $x^{\widetilde{\alpha}}=\int_{o}^{x} x^{\widetilde{\alpha}} \mathrm{d} x^{\mu}$ and $x^{\alpha}=\int_{\widetilde{o}}^{\widetilde{x}} x_{\widetilde{\mu}}^{\alpha} \mathrm{d} x^{\widetilde{\mu}}$. The numerical value of $x^{\widetilde{\alpha}}$ depends upon the detailed structure of the path $p$ from $o$ to $x$, and the numerical value of $x^{\alpha}$ depends upon the detailed structure of the path $\widetilde{p}$ from $\widetilde{o}$ to $\widetilde{x}$. There exist many paths $p$ which yield the same value of $x^{\widetilde{\alpha}}$, and many paths $\widetilde{p}$ which yield the same value of $x^{\alpha}$. This means that the correspondence between the points $x$ and $\widetilde{x}$ defined by the coordinates $x^{\alpha}$ and $x^{\widetilde{\alpha}}$ is both one-to-many and many-to-one, rather than one-to-one. However, there does exist a one-to-one correspondence between the paths $p$ and $\widetilde{p}$. Thus, if Eq. (11) is abandoned, the space is not a manifold, i.e., a space in which points are the most primitive geometrical (invariant) entities. It is instead a nonlocal space in which paths are the most primitive geometrical entities.

The fact that paths are our most primitive geometrical entities is interesting because the most intuitive description of quantum theory is Feynman's [6] path-integral formalism, and because a particle localized on a path is a string. Thus, it seems possible that if Eq. (1) is abandoned, this may lead serendipitously to what Witten [7] has called a much needed "conceptual, logical framework in which string theory is as natural as general relativity is in its terms."

Many investigators (see, e.g. Eddington [8]; Penrose, et. al. [9,10]; Finkelstein, et. al. [11-13]; Bergmann and Komar [14]; Gambini and Trias [15]) have expressed skepticism that a manifold adequately describes physical space. 
More recently and less formally, the same skepticism has been expressed by the Fields medalist Connes [16], and by Crane [17].

1.5. The Simplicity of Working in Path-Space. Let $\Phi$ be a pathdependent functional, i.e., a rule that assigns to each path $p$ a real number $\Phi(p)$. Following a method introduced by us [3] and independently by Mandelstam [18], we define derivatives of a path-dependent functional $\Phi(p)$ by giving the path $p$ an extension from its terminus $x$ while holding the rest of the path completely fixed. With this definition, one may work in pathspace almost as if it were a manifold. The main difference is that one must carefully preserve the orders of repeated partial derivatives. The following paragraph explains why this is so.

When we wish to emphasize the path-dependent character of a functional $\Phi$ we will use the notation $\Phi(p)$. However, our functionals include, as a subclass, the usual one-valued functions of $x^{\alpha}$, i.e., functionals which are "path-dependent" in the trivial sense that they depend only on the terminus $x$ of the path $p$; for them we use the notation $\Phi(x)$. From a path $p$ let two extended paths $p+\Delta p_{1}$ and $p+\Delta p_{2}$ be constructed such that the extensions $\Delta p_{1}$ and $\Delta p_{2}$ do not completely coincide, but such that the termini of $p+\Delta p_{1}$ and $p+\Delta p_{2}$ do coincide. The values of $\Phi\left(p+\Delta p_{1}\right)$ and $\Phi\left(p+\Delta p_{2}\right)$ are not generally equal. It follows that $\partial_{\mu} \partial_{\nu} \Phi$ and $\partial_{\nu} \partial_{\mu} \Phi$ are not generally equal. This may be seen by letting $\Delta p_{1}$ be an extension along which first only $x^{\mu}$ changes and then only $x^{\nu}$ changes, and letting $\Delta p_{2}$ be an extension along which first only $x^{\nu}$ changes and then only $x^{\mu}$ changes. Thus, we see that $\left[\partial_{\mu}, \partial_{\nu}\right] \Phi$ vanishes for functions $\Phi(x)$, but not generally for functionals $\Phi(p)$. The symbol $\left[\partial_{\mu}, \partial_{\nu}\right]=\partial_{\nu} \partial_{\mu}-\partial_{\mu} \partial_{\nu}$ denotes the usual commutator of partial derivatives.

\section{THE CONSERVATION GROUP}

In Pandres [19], we proposed that Eq. (1) be replaced by the weaker condition

$$
x_{\widetilde{\alpha}}^{\nu}\left(x_{\mu, \nu}^{\widetilde{\alpha}}-x_{\nu, \mu}^{\widetilde{\alpha}}\right)=0,
$$

and proved that this defines a group which contains the diffeomorphisms as a proper subgroup. This proof is given in Sec. 2.2. The covariance group defined by Eq. (41) is our "guiding principle," just as Einstein's requirement of covariance under the diffeomorphisms was the guiding principle for his theory of gravitation.

2.1. Justifications for Introducing the Conservation Group. Pauli [20] emphasized that "it is absolutely essential to insist that such a fundamental theorem as the covariance law should be derivable from the simplest 
possible basic assumptions." We now recall two (mathematically equivalent) basic assumptions which show that the covariance law based on Eq. (4) satisfies Pauli's requirement. The first of these assumptions, discussed in Sec. 2.1.1, explains why transformations that satisfy Eq. (4) are called "conservative."

2.1.1. Agreement Concerning Conserved Quantities. The first assumption [19] is that any two observers $\Omega$ and $\widetilde{\Omega}$, using the coordinates $x^{\alpha}$ and $x^{\widetilde{\alpha}}$, will agree whether or not a given quantity is conserved. A general relativistic conservation law is an expression of the form $V_{; \alpha}^{\alpha}=0$, where $V^{\alpha}$ is a vector density of weight +1 , and a semicolon denotes covariant differentiation with respect to the Christoffel symbol. We may however write this as

$$
V^{\alpha}{ }_{\alpha}=0
$$

because, for a vector density of weight +1 , the covariant divergence equals the ordinary divergence.

Eq. (5) is manifestly invariant under the diffeomorphisms. It is surprising, and very important, that Eq. (5) is also invariant under the larger group defined by Eq. (4). To see that this is true, we note that the transformation law for a vector density of weight +1 is

$$
V^{\widetilde{\alpha}}=\frac{\partial x}{\partial \widetilde{x}} x_{\mu}^{\widetilde{\alpha}} V^{\mu},
$$

where $\frac{\partial x}{\partial \widetilde{x}}$ is the determinant of $x^{\mu}{ }_{\widetilde{\alpha}}$. Upon differentiating Eq. (6) with respect to $x^{\widetilde{\alpha}}$, after a short calculation (Appendix), we obtain

$$
V_{, \widetilde{\alpha}}^{\widetilde{\alpha}}=\frac{\partial x}{\partial \widetilde{x}} V_{, \alpha}^{\alpha}+V^{\mu} \frac{\partial x}{\partial \widetilde{x}} x_{\widetilde{\alpha}}^{\nu}\left(x_{\mu, \nu}^{\widetilde{\alpha}}-x_{\nu, \mu}^{\widetilde{\alpha}}\right) .
$$

Since $\frac{\partial x}{\partial \widetilde{x}} \neq 0$, we see from Eq. (7) that, for arbitrary $V^{\mu}$, we have both $V^{\alpha}{ }_{\alpha}=0$ and $V^{\widetilde{\alpha}}, \widetilde{\alpha}=0$ if and only if the transformation between $x^{\alpha}$ and $x^{\widetilde{\alpha}}$ satisfies Eq. (4). For this reason, we call transformations that satisfy Eq. (4) "conservative."

\subsubsection{Agreement Concerning Equation for propagation of Light.}

The second assumption [21] is that any two observers will agree that the equation of motion for the propagation of light is the general relativistic wave equation $\frac{1}{\sqrt{-g}}\left(\sqrt{-g} g^{\alpha \nu} \Phi_{, \nu}\right),_{\alpha}=0$. Thus, our second assumption is that $\left(\sqrt{-g} g^{\alpha \nu} \Phi_{, \nu}\right), \alpha=0$ if and only if $\left(\sqrt{-\widetilde{g}} g^{\widetilde{\alpha} \widetilde{\nu}} \Phi_{, \widetilde{\nu}}\right), \widetilde{\alpha}=0$. This second assumption is mathematically equivalent to the first assumption since $\sqrt{-g} g^{\alpha \nu} \Phi_{, \nu}$ is a vector density of weight +1 . 
The second assumption is especially compelling because it is analogous to the step which led Einstein from special relativity to general relativity, and is also consistent with the assumption of constant light velocity that led him to special relativity. Thus, the group defined by Eq. (44) could be called the "light-propagation group." However, we will retain the name "conservation group," since this name has been used in all prior papers.

\subsection{Proof That Conservative Transformations Form a Group that} Contains the Diffeomorphisms as a Proper Subgroup. We now recall [19] an explicit proof that transformations which satisfy Eq. (4) form a group that contains the diffeomorphisms as a proper subgroup.

First, we note that the identity transformation $x^{\widetilde{\alpha}}=x^{\alpha}$ is a conservative transformation. Next, we consider the result of following a transformation from $x^{\alpha}$ to $x^{\widetilde{\alpha}}$ by a transformation from $x^{\widetilde{\alpha}}$ to $x^{\widehat{\alpha}}$. From the relation

$$
x_{\mu}^{\widehat{\alpha}}=x_{\widetilde{\rho}}^{\widehat{\alpha}} x_{\mu}^{\widetilde{\rho}}
$$

we find (Appendix) that

$$
x_{\widehat{\alpha}}^{\nu}\left(x_{\mu, \nu}^{\widehat{\alpha}}-x_{\nu, \mu}^{\widehat{\alpha}}\right)=x_{\widehat{\alpha}}^{\widetilde{\sigma}_{\widehat{\alpha}}}\left(x_{\widetilde{\rho}, \widetilde{\sigma}}^{\widehat{\alpha}}-x_{\widetilde{\sigma}, \widetilde{\rho}}^{\widehat{\alpha}}\right) x_{\mu}^{\widetilde{\rho}}+x_{\widetilde{\alpha}}^{\nu}\left(x_{\mu, \nu}^{\widetilde{\alpha}}-x_{\nu, \mu}^{\widetilde{\alpha}}\right) .
$$

We see from Eq. (9) that if $x_{\widetilde{\alpha}}^{\nu}\left(x_{\mu, \nu}^{\widetilde{\alpha}}-x_{\nu, \mu}^{\widetilde{\alpha}}\right)$ and $x^{\widetilde{\sigma}_{\widehat{\alpha}}}\left(x^{\widehat{\alpha}_{\tilde{\rho}}}, \widetilde{\sigma}-x^{\widehat{\alpha}}{ }_{\widetilde{\sigma}, \widetilde{\rho}}\right)$ vanish, then $x_{\widehat{\alpha}}^{\nu}\left(x_{\mu, \nu}^{\widehat{\alpha}}-x_{\nu, \mu}^{\widehat{\alpha}}\right)$ vanishes. This shows that if the transformations from $x^{\alpha}$ to $x^{\widetilde{\alpha}}$ and from $x^{\widetilde{\alpha}}$ to $x^{\widehat{\alpha}}$ are conservative, then the product transformation from $x^{\alpha}$ to $x^{\widehat{\alpha}}$ is conservative. If we let $x^{\widehat{\alpha}}=x^{\alpha}$, we see from Eq. (9) that the inverse of a conservative transformation is conservative. From Eq. (8), we see that the product of matrices $x^{\widetilde{\rho}}$ and $x_{\widetilde{\rho}}^{\widehat{\alpha}}$ (which represent the transformations from $x^{\alpha}$ to $x^{\widetilde{\alpha}}$ and from $x^{\widetilde{\alpha}}$ to $x^{\widehat{\alpha}}$, respectively) equals the matrix $x^{\widehat{\alpha}}{ }_{\mu}$ (which represents the product transformation from $x^{\alpha}$ to $x^{\widehat{\alpha}}$ ). It is obvious, and well known, that if products admit a matrix representation in this sense, then the associative law is satisfied. This completes the proof that the conservative transformations form a group.

We note that if Eq. (11) is satisfied, then Eq. (44) is satisfied; i.e., the conservation group contains the diffeomorphisms as a subgroup. Thus, to show that it contains the diffeomorphisms as a proper subgroup, we need only exhibit transformation coefficients which satisfy Eq. (44), but do not satisfy Eq. (1). It is easily verified (Appendix) that such transformation coefficients are

$$
\begin{aligned}
x^{\widetilde{\alpha}} & =\delta_{\mu}^{\alpha}+\delta_{0}^{\alpha} \delta_{\mu}^{2} x^{1} \\
x^{\nu}{ }_{\widetilde{\alpha}} & =\delta_{\alpha}^{\nu}-\delta_{0}^{\nu} \delta_{\alpha}^{2} x^{1}
\end{aligned}
$$

where $\delta_{\mu}^{\alpha}$ is the usual Kronecker delta. 


\section{THE CURVATURE VECTOR $C_{\mu}$}

We define $C_{\mu}$ by

$$
C_{\mu}=h_{i}^{\nu}\left(h_{\mu, \nu}^{i}-h_{\nu, \mu}^{i}\right)
$$

As we have noted in Section 1.1, Einstein [1] stated that introducing a richer structure than the metric would make it desirable to enlarge the covariance group. That the tetrad is such a richer structure may be seen as follows. If a definite tetrad $h_{\mu}^{i}$ is given, then the metric $g_{\mu \nu}$ is uniquely determined, but the converse is not true. If a definite metric $g_{\mu \nu}$ is given, then the tetrad $h_{\mu}^{i}$ is determined only up to a six-fold parameter of transformations, i.e., the homogeneous Lorentz transformations on the Latin (tetrad) indices.

We also note that no physics can be done without an observer and that a tetrad represents an observer-frame, as discussed by Synge [22]. Thus, a theory based on tetrads is especially parsimonious, and hence in accord with the philosophical principle called Occam's razor. It is also in accord with Dirac's [2] statement that the use of transformation theory "is very satisfactory from a philosophical point of view, as implying an increasing recognition of the part played by the observer in himself introducing the regularities that appear in his observations, and a lack of arbitrariness in the ways of nature ...."

Under a transformation from $x^{\alpha}$ to $x^{\widetilde{\alpha}}$, the tetrad transforms according to $h_{\mu}^{i}=h_{\widetilde{\alpha}}^{i} x^{\widetilde{\alpha}}$ and $h_{i}^{\nu}=h_{i}^{\widetilde{\alpha}} x_{\widetilde{\alpha}}^{\nu}$. Thus, we find (Appendix) that the transformation law for $C_{\mu}$ is

$$
C_{\mu}=C_{\widetilde{\alpha}} x_{\mu}^{\widetilde{\alpha}}+x_{\widetilde{\alpha}}^{\nu}\left(x_{\mu, \nu}^{\widetilde{\alpha}}-x_{\nu, \mu}^{\widetilde{\alpha}}\right),
$$

where $C_{\widetilde{\alpha}}=h_{i}^{\widetilde{\beta}}\left(h_{\widetilde{\alpha}, \widetilde{\beta}}^{i}-\left(h_{\widetilde{\beta}}^{i}, \widetilde{\alpha}\right)\right.$. We see from Eq. (12) that $C_{\mu}$ transforms as a vector if and only if the transformation is conservative. We now show that $C_{\mu}$ may be regarded as a curvature vector, because $C_{\mu}$ vanishes if and only if there exists a conservative transformation from $x^{\alpha}$ to a special $x^{\widetilde{\alpha}}$ in which $h_{\widetilde{\alpha}}^{i}$ is constant. This may be seen in the following way. If $h_{\widetilde{\alpha}}^{i}$ is constant then $C_{\widetilde{\alpha}}$ vanishes, so Eq. (12) shows that $C_{\mu}$ also vanishes if and only if the transformation is conservative. The converse is slightly less direct. Eq. (12) shows that any $C_{\mu}$ and $C_{\widetilde{\alpha}}$ which both vanish are related by a conservative transformation. Hence, if $C_{\mu}$ vanishes, then it is related by a conservative transformation to the $C_{\widetilde{\alpha}}$ with $h_{\widetilde{\alpha}}^{i}$ constant. Thus, we see that $C_{\mu}$ may be regarded as a curvature vector analogous, for the conservation group, to the Riemann curvature tensor $R_{\beta \mu \nu}^{\alpha}$ which, in the conventional (pathindependent) theory, vanishes if and only if there exists a diffeomorphism from $x^{\alpha}$ to a special $x^{\widetilde{\alpha}}$ in which $g_{\widetilde{\mu} \widetilde{\nu}}$ is constant. 
The definition given in Eq. (11) is only the first useful expression for $C_{\mu}$. There is a second expression, a third expression, and a fourth expression for $C_{\mu}$, which are sometimes useful

The second expression is obvious, i.e.,

$$
C_{\mu}=h_{i}^{\nu} f_{\nu \mu}^{i}
$$

where $f_{\mu \nu}^{i}=h_{\nu, \mu}^{i}-h_{\mu, \nu}^{i}$, as defined in Sec. 1.3.

We see from Eq. (11) that $C_{\mu}=h_{i}^{\nu}\left(h_{\mu, \nu}^{i}-h_{\nu, \mu}^{i}\right)=h_{i}^{\nu}\left(h_{\mu ; \nu}^{i}-h_{\nu ; \mu}^{i}\right)=$ $\gamma_{\mu \nu}^{\nu}-\gamma_{\nu \mu}^{\nu}$, where $\gamma_{i \mu \nu}=h_{i \mu ; \nu}$ is the Ricci rotation coefficient (Eisenhart [23]).

[The tetrad is used to convert between Greek (space-time) indices and Latin (tetrad) indices, e.g., $\gamma_{\alpha \mu j}=h_{\alpha}^{i} \gamma_{i \mu \nu} h_{j}^{\nu}$. Tetrad indices are raised and lowered by using $g^{i j}$ and $g_{i j}$, just as space-time indices are raised and lowered by using $g^{\mu \nu}$ and $g_{\mu \nu}$. Thus, for example $\gamma_{\mu \nu}^{i}=g^{i j} \gamma_{j \mu \nu}$, and $\gamma_{\mu \nu}^{\alpha}=h_{i}^{\alpha} \gamma_{\mu \nu}^{i}$.]

Now $\gamma_{\nu \mu}^{\nu}$ vanishes, because, as Eisenhart noted, the rotation coefficients are antisymmetric in their first two indices. Thus, from $C_{\mu}=\gamma_{\mu \nu}^{\nu}-\gamma_{\nu \mu}^{\nu}$, we have our third expression

$$
C_{\mu}=\gamma_{\mu \nu}^{\nu}
$$

The fourth expression will be given in Sec. 5.3.

The antisymmetry of $\gamma_{\mu \nu i}$ in $\mu$ and $\nu$ may be used (Appendix) to obtain an expression for $\gamma_{\mu \nu i}$ in terms of $f_{i \mu \nu}$. The result is

$$
\gamma_{\mu \nu i}=\frac{1}{2}\left(f_{i \mu \nu}-f_{\mu \nu i}-f_{\nu i \mu}\right) \text {. }
$$

\section{AN IDENTITY FOR THE EINSTEIN TENSOR}

Before recalling our theory which is covariant under the conservation group, it is convenient to obtain an identity for the Einstein tensor in terms of the Ricci rotation coefficients.

The Riemann curvature tensor is defined by

$$
R_{\beta \mu \nu}^{\alpha}=h_{i}^{\alpha}\left(h_{\beta ; \mu ; \nu}^{i}-h_{\beta ; \nu ; \mu}^{i}\right) \text {. }
$$

It is easily seen from the relation $\left(h_{i}^{\alpha} h_{\beta ; \mu}^{i}\right)_{; \nu}=h_{i}^{\alpha} h_{\beta ; \mu ; \nu}^{i}+h_{i ; \nu}^{\alpha} h_{\beta ; \mu}^{i}$ that the expression for $R_{\beta \mu \nu}^{\alpha}$ may be manipulated into the form

$$
R_{\beta \mu \nu}^{\alpha}=\gamma_{\beta \mu ; \nu}^{\alpha}-\gamma_{\beta \nu ; \mu}^{\alpha}+\gamma_{\sigma \nu}^{\alpha} \gamma_{\beta \mu}^{\sigma}-\gamma_{\sigma \mu}^{\alpha} \gamma_{\beta \nu}^{\sigma}
$$

which is the well known Ricci identity for the curvature tensor (see, e.g., Taub [24]) in terms of the Ricci rotation coefficients.

The Einstein tensor is defined as usual by $G_{\mu \nu}=R_{\mu \nu}-\frac{1}{2} g_{\mu \nu} R$, where $R_{\mu \nu}=R_{\mu \alpha \nu}^{\alpha}$ is the usual Ricci tensor, and $R=R_{\alpha}^{\alpha}$ is the usual Ricci scalar. From Eq. (16) and Eq. (14), it follows easily that identities for the Ricci tensor and the Ricci scalar are

$$
R_{\mu \nu}=C_{\mu ; \nu}-C_{\alpha} \gamma_{\mu \nu}^{\alpha}+\gamma_{\mu \nu ; \alpha}^{\alpha}+\gamma_{\sigma \nu}^{\alpha} \gamma_{\mu \alpha}^{\sigma}
$$


and

$$
R=2 C_{; \alpha}^{\alpha}+C^{\alpha} C_{\alpha}-\gamma^{\alpha \sigma \beta} \gamma_{\beta \sigma \alpha} .
$$

Thus, our identity for the Einstein tensor is

$$
\begin{aligned}
G_{\mu \nu}= & C_{\mu ; \nu}-C_{\alpha} \gamma_{\mu \nu}^{\alpha}-g_{\mu \nu} C_{; \alpha}^{\alpha}-\frac{1}{2} g_{\mu \nu} C^{\alpha} C_{\alpha} \\
& +\gamma_{\mu \nu ; \alpha}^{\alpha}+\gamma_{\sigma \nu}^{\alpha} \gamma_{\mu \alpha}^{\sigma}+\frac{1}{2} g_{\mu \nu} \gamma^{\alpha \sigma \beta} \gamma_{\beta \sigma \alpha} .
\end{aligned}
$$

\section{THEORY COVARIANT UNDER THE CONSERVATION GROUP}

5.1. The Lagrangian. In [19], we chose $g^{\mu \nu} C_{\mu} C_{\nu}$ as our Lagrangian because it is the only invariant that can be formed from the curvature vector $C_{\mu}$ by contraction. Thus, we used the variational principle

$$
\delta \int g^{\mu \nu} C_{\mu} C_{\nu} \sqrt{-g} d^{4} x=0
$$

where the tetrad $h_{j}{ }^{\nu}$ is varied.

A tedious, but straightforward, calculation (Appendix) shows that this variational principle leads to the field equations

$$
C_{\mu ; \nu}-C_{\alpha} \gamma_{\mu \nu}^{\alpha}-g_{\mu \nu} C_{; \alpha}^{\alpha}-\frac{1}{2} g_{\mu \nu} C^{\alpha} C_{\alpha}=0 \text {. }
$$

Notice that Eq. (21) just states that the first line in Eq. (19), the identity for the Einstein tensor, vanishes. Thus, we may write our field equations in the form

$$
G_{\mu \nu}=\gamma_{\mu \nu ; \alpha}^{\alpha}+\gamma_{\sigma \nu}^{\alpha} \gamma_{\mu \alpha}^{\sigma}+\frac{1}{2} g_{\mu \nu} \gamma^{\alpha \sigma \beta} \gamma_{\beta \sigma \alpha}
$$

The variational calculation in the Appendix also shows that Noether's theorem yields the six currents

$$
\mathfrak{J}_{i j}{ }^{\nu}=C_{\alpha}\left(h_{i}^{\alpha} h_{j}^{\nu}-h_{j}^{\alpha} h_{i}^{\nu}\right)
$$

which satisfy the conservation laws $\mathfrak{J}_{i j: \nu}^{\nu}=0$. Indeed, the antisymmetric part of our field equations, Eq. (21), just states that $\mathfrak{J}_{i j: \nu}^{\nu}=0$. It is, however, the symmetric part of the field equations which will display the unification of the gravitational and electroweak fields.

5.2. Schrödinger's Interpretation of the Einstein Equations. The Einstein equations of general relativity $G_{\mu \nu}=T_{\mu \nu}$ relate a geometrical object, the Einstein tensor $G_{\mu \nu}$ on the left, to a physical object, the stress-energy tensor $T_{\mu \nu}$ on the right. Einstein felt that this was undesirable and that the use of $T_{\mu \nu}$ in this way was at best a provisional solution.

Schrödinger [25] proposed an attractive general solution to this problem. He suggested that the Einstein equations should be regarded not as differential equations to be solved for $g_{\mu \nu}$, with $T_{\mu \nu}$ given, but rather as the definition 
of the stress-energy tensor: "I would rather that you did not regard these equations as field equations, but rather as a definition of $T_{i k}$, the matter tensor."

In our theory, however, this is not quite satisfactory, because the right-hand side of our Eq. (22) for $G_{\mu \nu}$ in not generally symmetric in $\mu$ and $\nu$. This is not a contradiction because, as we have stated in Sec. 1.5, when quantities are path-dependent, one must carefully preserve the orders of repeated partial derivatives. Taking this into account, one finds (Appendix) that

$$
G_{\mu \nu}-G_{\nu \mu}=h_{i}^{\alpha}\left(\left[\partial_{\nu}, \partial_{\alpha}\right] h_{\mu}^{i}+\left[\partial_{\alpha}, \partial_{\mu}\right] h_{\nu}^{i}+\left[\partial_{\mu}, \partial_{\nu}\right] h_{\alpha}^{i}\right) \quad .
$$

Thus, $G_{\mu \nu}$ is not generally symmetric if the tetrad is path-dependent. (We suspect that any tetrad which satisfies the field equations admits a conservative transformation to a coordinate system in which it is path-independent, but we have not succeeded in proving this.)

The stress-energy tensor $T_{\mu \nu}$, however, must be symmetric in order to have the needed physical properties. We therefore adopt a slight modification of Schrödinger's proposal, in which we define $T_{\mu \nu}=G_{\mu \nu}$, where $G_{\mu \nu}=\frac{1}{2}\left(G_{\mu \nu}+\right.$ $\left.G_{\nu \mu}\right)$ is the symmetric part of $G_{\mu \nu}$. Thus, from Eq. (22), we obtain

$$
T_{\mu \nu}=\frac{1}{2}\left(\gamma_{\mu \nu ; \alpha}^{\alpha}+\gamma_{\nu \mu ; \alpha}^{\alpha}+\gamma_{\sigma \nu}^{\alpha} \gamma_{\mu \alpha}^{\sigma}+\gamma_{\sigma \mu}^{\alpha} \gamma_{\nu \alpha}^{\sigma}+g_{\mu \nu} \gamma^{\alpha \sigma \beta} \gamma_{\beta \sigma \alpha}\right) .
$$

5.3. Physical Interpretation for the Stress-Energy Tensor. In order to manipulate Eq. (24) into a form such that the physical interpretation of $T_{\mu \nu}$ is clear, we have used [26] the permutation group decomposition of the Ricci rotation coefficient $\gamma_{\mu \nu i}$.

The permutation group on three indices has six group elements. One element is the identity. The other five group elements are "cycles" such as ( $\mu \nu i)$, which has the effect of replacing $\mu$ with $\nu, \nu$ with $i$, and $i$ with $\mu$. These five group elements are $(\mu \nu),(\nu i),(i \mu),(\mu i \nu)$, and $(\mu \nu i)$.

We have

$$
\gamma_{\mu \nu i}=A_{\mu \nu i}+M_{\mu \nu i},
$$

where $A_{\mu \nu i}$ is the totally antisymmetric part, and $M_{\mu \nu i}$ is the "mixed symmetry" part of $\gamma_{\mu \nu i}$. (The totally symmetric part vanishes because $\gamma_{\mu \nu i}$ is antisymmetric in its first two indices.)

The totally antisymmetric part is

$$
A_{\mu \nu i}=\frac{1}{3}\left(\gamma_{\mu \nu i}+\gamma_{\nu i \mu}+\gamma_{i \mu \nu}\right) \text {. }
$$

Hence, the mixed symmetry part is $M_{\mu \nu i}=\gamma_{\mu \nu i}-A_{\mu \nu i}$. This gives

$$
M_{\mu \nu i}=\frac{1}{3}\left(2 \gamma_{\mu \nu i}-\gamma_{\nu i \mu}-\gamma_{i \mu \nu}\right)=\frac{1}{3}\left(2 \gamma_{\mu \nu i}+\gamma_{i \nu \mu}-\gamma_{i \mu \nu}\right),
$$

which is antisymmetric in $\mu$ and $\nu$. We shall see (as discussed in [27]) that $M_{\mu \nu i}$ describes the electroweak field. 
We see from Eq. (14) and Eq. (25) that

$$
C_{\mu}=M_{\mu \nu}^{\nu}
$$

which is our fourth expression for $C_{\mu}$.

We also need a vector $A^{\mu}$ which is essentially the dual of $A_{\alpha \beta \sigma}$, i.e.,

$$
A^{\mu}=\frac{1}{3 !}(-g)^{-1 / 2} e^{\mu \alpha \beta \sigma} A_{\alpha \beta \sigma} \quad .
$$

where $e^{\mu \alpha \beta \sigma}$ is the usual Levi-Civita tensor density of weight +1 .

If we substitute Eq. (25) into Eq. (24), a tedious calculation (Appendix) gives

$$
\begin{aligned}
T_{\mu \nu}= & \frac{1}{2}\left(J_{\mu i} h_{\nu}^{i}+J_{\nu i} h_{\mu}^{i}\right)-\left(M_{\mu i}^{\alpha} M_{\alpha \nu}{ }^{i}-\frac{1}{4} g_{\mu \nu} M^{\alpha \sigma i} M_{\alpha \sigma i}\right) \\
& +2 A_{\mu} A_{\nu}+g_{\mu \nu} A^{\alpha} A_{\alpha},
\end{aligned}
$$

where the $J_{\mu i}=M_{\mu i ; \alpha}^{\alpha}$ are the currents which are the source of the electroweak field $M_{\mu \nu i}$. (If the tetrad is path-dependent, this could explain the experimentally observed electroweak parity breaking, and we note that in the Weinberg-Salam theory this parity breaking is "put in by hand."

The quantity

$$
M_{\mu \nu}=M_{\mu i}^{\alpha} M_{\alpha \nu}^{i}-\frac{1}{4} g_{\mu \nu} M^{\alpha \sigma i} M_{\alpha \sigma i}
$$

has the formal structure of the stress-energy tensor for the Salam [28] Weinberg [29] electroweak theory, and Eq. (30) contains the terms $2 A_{\mu} A_{\nu}+$ $g_{\mu \nu} A^{\alpha} A_{\alpha}$ that correspond to the presence of mass.

It is important to note that the quantity $M_{\mu \nu}^{i}$ appears in our equations because the diffeomorphisms have been replaced by the larger conservation group. There is no need to introduce $M_{\mu \nu}{ }^{i}$ as a "compensating field" corresponding to a $U(1) \times S U(2)$ gauge group, as Yang and Mills [30] did in their original paper on gauge theory, motivated by Heisenberg's idea that if the electromagnetic field were "turned off" it would be impossible to distinguish between a proton and a neutron. There is also no need to depend upon spontaneous symmetry breaking to bring in Higgs bosons and mass. Moreover, it is clear that Pauli's requirement that the covariance law be derived from the simplest possible basic assumptions is met less satisfactorily by the assumptions that justify gauge theory than by either our assumption that all observers will agree whether a given quantity is conserved, or our assumption that all observers will agree that the propagation of light is described by the general relativistic wave equation.

Equation (31) is manifestly invariant under homogeneous Lorentz transformations on the Latin indices. However, these transformations mimic those of the $U(1) \times S U(2)$ gauge group. This may be seen in the following way. 
We find from Eq. (15) and Eq. (27) that

$$
M_{\mu \nu i}=\frac{1}{3}\left(2 f_{i \mu \nu}-f_{\mu \nu i}-f_{\nu i \mu}\right),
$$

which may be written

$$
M_{\mu \nu i}=\frac{1}{3}\left(2 \delta_{i}^{n} \delta_{\mu}^{\alpha} \delta_{\nu}^{\sigma}-h_{\mu}^{n} \delta_{\nu}^{\alpha} h_{i}^{\sigma}-h_{\nu}^{n} h_{i}^{\alpha} \delta_{\mu}^{\sigma}\right) f_{n \alpha \sigma}
$$

It is interesting, and perhaps important, to notice that Eq. (33) may be rewritten into the form

$$
M_{\mu \nu i}=\frac{1}{3}\left(2 \delta_{i}^{n} \delta_{\mu}^{\alpha} \delta_{\nu}^{\sigma}-h_{\mu}^{n} \delta_{\nu}^{\alpha} h_{i}^{\sigma}-h_{\nu}^{n} h_{i}^{\alpha} \delta_{\mu}^{\sigma}\right) \mathfrak{F}_{n \alpha \sigma}
$$

where

$$
\mathfrak{F}_{n \alpha \sigma}=f_{n \alpha \sigma}+e_{0 n j k} h_{\alpha}^{j} h_{\sigma}^{k}
$$

is the usual field strength (see, e.g., Nakahara [31]) for a $U(1) \times S U(2)$ gauge field. In rewriting Eq. (33) as Eq. (34), we have used the easily verifiable fact that

$$
\left(2 \delta_{i}^{n} \delta_{\mu}^{\alpha} \delta_{\nu}^{\sigma}-h_{\mu}^{n} \delta_{\nu}^{\alpha} h_{i}^{\sigma}-h_{\nu}^{n} h_{i}^{\alpha} \delta_{\mu}^{\sigma}\right) e_{0 n j k} h_{\alpha}^{j} h_{\sigma}^{k}=0 \quad .
$$

From Eq. (34), we see that in the expression, Eq. (33), for $M_{\mu \nu i}$, the curl $f_{n \alpha \sigma}$ may simply be replaced by the gauge field strength $\mathfrak{F}_{n \alpha \sigma}$. In our theory, the quantity $\mathfrak{F}_{n \alpha \sigma}$ does not directly describe the electroweak field. It is, however, the fundamental quantity which is essential for the construction of that field. The $\mathfrak{F}_{n \alpha \sigma}$ in Eq. (34) may be viewed as a field with "bare" or massless quanta, which is "clothed" by the factor $\frac{1}{3}\left(2 \delta_{i}^{n} \delta_{\mu}^{\alpha} \delta_{\nu}^{\sigma}-h^{n}{ }_{\mu} \delta_{\nu}^{\alpha} h_{i}^{\sigma}-h_{\nu}^{n} h_{i}^{\alpha} \delta_{\mu}^{\sigma}\right)$. It is $M_{\mu \nu i}$ that we identify as the physical electroweak field, and which appears in the appropriate way in the stress-energy tensor of the Einstein equations. For this identification to be valid, the quantity $M_{\mu \nu 0}=\frac{1}{3}\left(2 f_{0 \mu \nu}-f_{\mu \nu 0}-f_{\nu 0 \mu}\right)$ must describe the electromagnetic field; hence, it must be the curl of a vector. The presence of the terms $-f_{\mu \nu 0}-f_{\nu 0 \mu}$ may cause one to ask how $M_{\mu \nu 0}$ can be identified as the electromagnetic field.

Our answer is this: The orthodox physical interpretation, which we adopt, is that $h_{\mu}^{i}$ describes an observer-frame. Now, if $h_{\mu}^{i}$ describes a freely-falling, nonrotating observer frame, our expression for $M_{\mu \nu 0}$ reduces to $M_{\mu \nu 0}=\frac{1}{3} f_{0 \mu \nu}$. This may be seen as follows. The condition for a freely-falling, nonrotating frame (Synge [22]) is $h_{i \nu ; \alpha} h_{0}^{\alpha}=0$. In terms of the Ricci rotation coefficients, the condition is $\gamma_{\mu \nu 0}=0$. From this and Eq. (27), we see that for an $h_{\mu}^{i}$ which describes a freely falling, nonrotating observer frame, $M_{\mu \nu 0}=\frac{1}{3}\left(\gamma_{0 \nu \mu}-\gamma_{0 \mu \nu}\right)=\frac{1}{3}\left(h_{0 \nu ; \mu}-h_{0 \mu ; \nu}\right)=\frac{1}{3}\left(h_{0 \nu, \mu}-h_{0 \mu}, \nu\right)=\frac{1}{3} f_{0 \mu \nu}$. 
5.4. Further Insight Into the Lagrangian. In retrospect, we can now see intuitively why the Lagrangian $g^{\mu \nu} C_{\mu} C_{\nu}=C^{\alpha} C_{\alpha}$ yields a theory which unifies the gravitational and electroweak interactions, and includes a term that brings in mass.

We see from Eq. (18) that

$$
C^{\alpha} C_{\alpha}=R+\gamma^{\mu i \nu} \gamma_{\mu \nu i}-2 C_{; \alpha}^{\alpha}
$$

The first term on the right side of Eq. (36) is the Ricci scalar, which is the Lagrangian for gravitation. The last term is a covariant divergence, which contributes nothing to the field equations. After a bit of work (Appendix), we obtain $\gamma^{\mu i \nu} \gamma_{\mu \nu i}=\frac{1}{2} M^{\mu \nu i} M_{\mu \nu i}+6 A^{\alpha} A_{\alpha}$, and our expression for the Lagrangian becomes

$$
C^{\alpha} C_{\alpha}=R+\frac{1}{2} M^{\mu \nu i} M_{\mu \nu i}+6 A^{\alpha} A_{\alpha}-2 C_{; \alpha}^{\alpha} .
$$

The term $M^{\mu \nu i} M_{\mu \nu i}$ is in the form of the usual electroweak Lagrangian, and the $A^{\alpha} A_{\alpha}$ term has precisely the form that is needed (see, e.g., Moriyasu [32]) for the introduction of mass.

5.5. Concerning Quantization. As many investigators agree, a major reason for the difficulty (perhaps impossibility) in quantizing general relativity is that the Lagrangian $R$ contains higher than first derivatives of the metric. By contrast, the Lagrangian $C^{\alpha} C_{a}$ contains no higher than first derivatives of the tetrad. Also, $C^{\alpha} C_{a}$ is only quadratic in these first derivatives. We are therefore optimistic that quantization of the theory with Lagrangian $C^{\alpha} C_{a}$ may be much more straightforward than the theory with Lagrangian $R$. Previously [33], we have used the Dirac-Bergmann algorithm (Bergmann and Goldberg [34], Dirac [35], Sundermeyer [36], Weinberg [37]) for constrained dynamics to find the consistent Hamiltonian for this theory.

\section{CONCLUSIONS}

Einstein introduced the diffeomorphisms in order to accommodate observers who are accelerated with respect to one another. But, is there a need for the inclusion of some still more general class of observers? In this Section, we recall a somewhat speculative argument [38] that there is such a need.

General relativity makes use of a classical observer who can observe the motion of a physical system without disturbing the system. This violates the fundamental principles of quantum theory. Most discussions of observation in quantum theory make use of a "macroscopic" classical observer - one who can "stand outside" a quantum mechanical system and act upon the system without being acted upon by the system. This is unsatisfactory, because 
there exist no observers who are infinitely large by comparison with quantum mechanical systems. One solution of this problem was given by Everett [39]. He considers a quantum observer's memory to have quantum states that are correlated with the states of what he has observed. Each observer can then consider himself a macroscopic observer (since his different states are independent) and still treat other observers as part of his quantum mechanical universe. Each observer can also assign coordinates to events in his universe. The uncertainty principle does not limit the precision with which he can do this, because the four operators which represent his coordinates commute. Let $\Omega$ and $\widetilde{\Omega}$ be two observers, each of whom considers himself a macroscopic observer, while treating the other as part of his quantum mechanical universe. Let $x^{\alpha}$ and $x^{\widetilde{\alpha}}$ be coordinates which are assigned by $\Omega$ and $\widetilde{\Omega}$, respectively, to events in their universes. The assumption that physical space-time is a differentiable manifold rests squarely upon the assertion that it is possible to establish a one-to-one correspondence between $x^{\alpha}$ and $x^{\widetilde{\alpha}}$, at least in coordinate patches. Einstein challenged the validity of Newton's absolute time on the ground that no operational method had been, or could be, given for its measurement. In this spirit, we challenge the validity of space-time being a differentiable manifold, on the ground that no operational measurement has been, or can be, given for establishing a one-to-one correspondence between the coordinates $x^{\alpha}$ and $x^{\widetilde{\alpha}}$. Our two observers are free to exchange information so that, for example, $\Omega$ can possess a complete description of the procedure which $\widetilde{\Omega}$ uses in assigning coordinates to events. If $\Omega$ could also state with certainty (as in general relativity) that $\widetilde{\Omega}$ 's world-line is a particular path $p$, then he could write an expression uniquely specifying $\widetilde{\Omega}$ 's coordinates $x^{\widetilde{\alpha}}$ in terms of his own coordinates $x^{\alpha}$. Thus $x^{\widetilde{\alpha}}$ could be regarded as a functional of the known path $p$ with terminus $x$ having the coordinates $x^{\alpha}$, i.e., $x^{\widetilde{\alpha}}=x^{\widetilde{\alpha}}(x)$. However, the observer $\Omega$ has described $\widetilde{\Omega}$ 's world-line as completely as nature permits when he states that all paths occur with equal probability amplitude, in the sense that the probability amplitude for a path $p$ is $N e^{i L(p) / \hbar}$ where $L$ is $\Omega$ 's Lagrangian for $\widetilde{\Omega}, N$ is a normalization factor (the same for all paths), and $\hbar$ is the usual quantum of angular momentum. Therefore, $\Omega$ can only state that the probability amplitude for $\widetilde{\Omega}$ 's coordinate number $x^{\widetilde{\alpha}}$ corresponding to his coordinate number $x^{\alpha}$ is

$$
\Psi\left(x^{\alpha}, x^{\widetilde{\alpha}}\right)=\sum_{p} N e^{i L(p) / \hbar}
$$

where $\sum_{p}$ denotes the democratic sum with equal weight of contributions due to all paths $p$ with terminus $x^{\alpha}$ such that the image-paths $\widetilde{p}$ have terminus $x^{\widetilde{\alpha}}$. As the sizes of observers $\Omega$ and $\widetilde{\Omega}$ increase without limit, we find that 
the competing alternatives in equation (38) interfere destructively on all but the classically allowed path. Thus Eq. (38) goes over to the transformation $x^{\widetilde{\alpha}}=x^{\widetilde{\alpha}}(x)$, with the inverse transformation $x^{\alpha}=x^{\alpha}(\widetilde{x})$, in the macroscopic limit.

The above interpretation that conservative transformations relate the measurements of "quantum observers" is attractive, but is not essential for our unification of the gravitational and electroweak interactions.

We have obtained our unification by following Einstein's [1] general suggestion that the diffeomorphisms be replaced by some larger group, i.e., some appropriate covariance group that contains the diffeomorphisms as a proper subgroup. We have obtained such an appropriate group, the conservation group, by assuming that all observers will agree whether or not any given quantity is conserved. Field equations have been obtained from a variational principle with a Lagrangian invariant under the conservation group. (Our Lagrangian is analogous to the conventional Lagrangian $R$ invariant under the diffeomorphisms, used in deriving Einstein's equations for gravitation alone.) Our field equations unify the gravitational and electroweak interactions. The gravitational field is described by the metric, as in general relativity. The electroweak field is described by the "mixed symmetry" part of the Ricci rotation coefficients under the permutation group on three indices. Our theory has a stress-energy tensor which agrees with that of the Salam-Weinberg electroweak theory, and contains terms that correspond to the presence of mass.

\section{APPENDIX}

In this Appendix, we give the detailed computation justifying certain equations in order to optimize readability.

Equations (2) and (3). From $\mathrm{d} x^{i}=h_{\mu}^{i} \mathrm{~d} x^{\mu}$, we have $\frac{\mathrm{d} x^{i}}{\mathrm{~d} s}=h_{\mu}^{i} \frac{\mathrm{d} x^{\mu}}{\mathrm{d} s}$, so

$$
\frac{\mathrm{d}^{2} x^{i}}{\mathrm{~d} s^{2}}=h_{\mu}^{i} \frac{\mathrm{d}^{2} x^{\mu}}{\mathrm{d} s^{2}}+\left(\frac{\mathrm{d}}{\mathrm{d} s} h_{\mu}^{i}\right) \frac{\mathrm{d} x^{\mu}}{\mathrm{d} s}=h_{\mu}^{i} \frac{\mathrm{d}^{2} x^{\mu}}{\mathrm{d} s^{2}}+h_{\mu, \nu}^{i} \frac{\mathrm{d} x^{\mu}}{\mathrm{d} s} \frac{\mathrm{d} x^{\nu}}{\mathrm{d} s} .
$$

Thus, since $\frac{\mathrm{d}^{2} x^{i}}{\mathrm{~d} s^{2}}=0$, multiplication by $h_{i}^{\alpha}$ gives

$$
\frac{\mathrm{d}^{2} x^{\alpha}}{\mathrm{d} s^{2}}+h_{i}^{\alpha} h_{\mu, \nu}^{i} \frac{\mathrm{d} x^{\mu}}{\mathrm{d} s} \frac{\mathrm{d} x^{\nu}}{\mathrm{d} s}=0
$$

We need an alternative expression for $h_{i}^{\alpha} h_{\mu, \nu}^{i}$. The Christoffel symbol is

$$
\Gamma_{\mu \nu}^{\alpha}=\frac{1}{2} g^{\alpha \beta}\left(g_{\beta \mu, \nu}+g_{\beta \nu, \mu}-g_{\mu \nu, \beta}\right)
$$


From $g_{\mu \nu}=g_{i j} h_{\mu}^{i} h_{\nu}^{j}$, we get $g_{\mu \nu, \beta}=g_{i j} h_{\mu, \beta}^{i} h_{\nu}^{j}+g_{i j} h_{\mu}^{i} h_{\nu, \beta}^{j}$, so

$$
\begin{aligned}
g_{\beta \mu, \nu}+g_{\beta \nu, \mu}-g_{\mu \nu, \beta} & =g_{i j}\left(h_{\mu}^{i} h_{\beta, \nu}^{j}+h_{\beta}^{i} h_{\mu, \nu}^{j}+h_{\nu}^{i} h_{\beta, \mu}^{j}+h_{\beta}^{i} h_{\nu, \mu}^{j}-h_{\nu}^{i} h_{\mu, \beta}^{j}-h_{\mu}^{i} h_{\nu, \beta}^{j}\right) \\
& =2 g_{i j} h_{\beta}^{i} h_{\mu, \nu}^{j}+g_{i j}\left(h_{\beta}^{i} f_{\mu \nu}^{j}-h_{\mu}^{i} f_{\beta \nu}^{j}-h_{\nu}^{i} f_{\beta \mu}^{j}\right) .
\end{aligned}
$$

If we multiply the above equation by $\frac{1}{2} g^{\alpha \beta}$ and use Eq. (40), we get

$$
h_{i}^{\alpha} h_{\mu, \nu}^{i}=\Gamma_{\mu \nu}^{\alpha}-\frac{1}{2} g^{\alpha \beta} g_{i j}\left(h_{\beta}^{i} f_{\mu \nu}^{j}-h_{\mu}^{i} f_{\beta \nu}^{j}-h_{\nu}^{i} f_{\beta \mu}^{j}\right) .
$$

We multiply this equation by $\frac{\mathrm{d} x^{\mu}}{\mathrm{d} s} \frac{\mathrm{d} x^{\nu}}{\mathrm{d} s}$, to get

$$
h_{i}^{\alpha} h_{\mu, \nu}^{i} \frac{\mathrm{d} x^{\mu}}{\mathrm{d} s} \frac{\mathrm{d} x^{\nu}}{\mathrm{d} s}=\Gamma_{\mu \nu}^{\alpha} \frac{\mathrm{d} x^{\mu}}{\mathrm{d} s} \frac{\mathrm{d} x^{\nu}}{\mathrm{d} s}+g^{\alpha \beta} g_{i j} h_{\mu}^{i} f_{\beta \nu}^{j} \frac{\mathrm{d} x^{\mu}}{\mathrm{d} s} \frac{\mathrm{d} x^{\nu}}{\mathrm{d} s} .
$$

We use this expression for $h_{i}^{\alpha} h_{\mu, \nu}^{i} \frac{\mathrm{d} x^{\mu}}{\mathrm{d} s} \frac{\mathrm{d} x^{\nu}}{\mathrm{d} s}$ in Eq. (39) to obtain

$$
\frac{\mathrm{d}^{2} x^{\alpha}}{\mathrm{d} s^{2}}+\Gamma_{\mu \nu}^{\alpha} \frac{\mathrm{d} x^{\mu}}{\mathrm{d} s} \frac{\mathrm{d} x^{\nu}}{\mathrm{d} s}=-g^{\alpha \beta} g_{i j} h_{\mu}^{i} \frac{\mathrm{d} x^{\mu}}{\mathrm{d} s} f_{\beta \nu}^{j} \frac{\mathrm{d} x^{\nu}}{\mathrm{d} s} .
$$

Notice that $h_{\mu}^{i} \frac{\mathrm{d} x^{\mu}}{\mathrm{d} s}=\frac{\mathrm{d} x^{i}}{\mathrm{~d} s}$, which is the (constant) first integral of the free particle equation $\frac{\mathrm{d}^{2} x^{i}}{\mathrm{~d} s^{2}}=0$. We denote $h_{\mu}^{i} \frac{\mathrm{d} x^{\mu}}{\mathrm{d} s}=\frac{\mathrm{d} x^{i}}{\mathrm{~d} s}$ by $v^{i}$, and see that Eq. (41) becomes

$$
\frac{\mathrm{d}^{2} x^{\alpha}}{\mathrm{d} s^{2}}+\Gamma_{\mu \nu}^{\alpha} \frac{\mathrm{d} x^{\mu}}{\mathrm{d} s} \frac{\mathrm{d} x^{\nu}}{\mathrm{d} s}=-g^{\alpha \beta} v_{i} f_{\beta \nu}^{i} \frac{\mathrm{d} x^{\nu}}{\mathrm{d} s} .
$$

Finally, we define $f_{\mu \nu}=v_{i} f_{\mu \nu}^{i}$, and see that the above equation becomes

$$
\frac{\mathrm{d}^{2} x^{\alpha}}{\mathrm{d} s^{2}}+\Gamma_{\mu \nu}^{\alpha} \frac{\mathrm{d} x^{\mu}}{\mathrm{d} s} \frac{\mathrm{d} x^{\nu}}{\mathrm{d} s}=-f_{\nu}^{\alpha} \frac{\mathrm{d} x^{\nu}}{\mathrm{d} s}
$$

which is our Eq. (3).

Equations (6) and (7). The transformation law for a vector density of weight +1 is our Eq. (6)

$$
V^{\widetilde{\alpha}}=\frac{\partial x}{\partial \widetilde{x}} x_{\mu}^{\widetilde{\alpha}} V^{\mu},
$$

Upon differentiating Eq. ([6) with respect to $x^{\widetilde{\alpha}}$, we have

$$
V^{\widetilde{\alpha}}, \widetilde{\alpha}=\frac{\partial x}{\partial \widetilde{x}} V^{\alpha},{ }_{\alpha}+V^{\mu}\left(\frac{\partial x}{\partial \widetilde{x}} x_{\mu}^{\widetilde{\alpha}}\right), \widetilde{\alpha} .
$$

Now,

$$
\begin{aligned}
\left(\frac{\partial x}{\partial \widetilde{x}} x_{\mu}^{\widetilde{\alpha}}\right)_{, \widetilde{\alpha}} & =\left(\frac{\partial x}{\partial \widetilde{x}}\right),{ }_{\widetilde{\alpha}} x^{\widetilde{\alpha}}+\frac{\partial x}{\partial \widetilde{x}} x_{\mu, \widetilde{\alpha}}^{\widetilde{\alpha}}=\left(\frac{\partial x}{\partial \widetilde{x}}\right)_{, \mu}+\frac{\partial x}{\partial \widetilde{x}} x_{\mu, \nu}^{\widetilde{\alpha}} x_{\widetilde{\alpha}}^{\nu} \\
& =\frac{\partial x}{\partial \widetilde{x}} x^{\nu}{ }_{\widetilde{\alpha}}\left(x_{\mu, \nu}^{\widetilde{\alpha}}-x^{\widetilde{\alpha}}{ }_{\nu, \mu}\right), \\
& 17
\end{aligned}
$$


where we have used the well-known formula $\left(\frac{\partial x}{\partial \widetilde{x}}\right)_{, \mu}=\frac{\partial x}{\partial \widetilde{x}} x_{\nu}^{\widetilde{\alpha}} x_{\widetilde{\alpha}, \mu}$ for the derivative of a determinant. Upon substituting the expression $\left(\frac{\partial x}{\partial \widetilde{x}} x^{\widetilde{\alpha}}{ }_{\mu}\right), \widetilde{\alpha}=$ $\frac{\partial x}{\partial \widetilde{x}} x_{\widetilde{\alpha}}^{\nu}\left(x_{\mu, \nu}^{\widetilde{\alpha}}-x_{\nu, \mu}^{\widetilde{\alpha}}\right)$ into Eq. (42), we obtain our Eq. (7),

$$
V_{, \widetilde{\alpha}}^{\widetilde{\alpha}}=\frac{\partial x}{\partial \widetilde{x}} V^{\alpha}{ }_{\alpha}+V^{\mu} \frac{\partial x}{\partial \widetilde{x}} x_{\widetilde{\alpha}}^{\nu}\left(x_{\mu, \nu}^{\widetilde{\alpha}}-x_{\nu, \mu}^{\widetilde{\alpha}}\right) \text {. }
$$

Equations (8) and (9). Upon differentiating Eq. (8), $x^{\widehat{\alpha}}{ }_{\mu}=x^{\widehat{\alpha}_{\widetilde{\rho}}} x^{{ }^{\widetilde{\rho}}}$ with respect to $x^{\nu}$, we get

$$
x_{\mu, \nu}^{\widehat{\alpha}}=x_{\widetilde{\rho}, \nu}^{\widehat{\alpha}} x_{\mu}^{\widetilde{\rho}}+x_{\widetilde{\rho}}^{\widehat{\alpha}} x_{\mu, \nu}^{\widetilde{\rho}}=x_{\widetilde{\rho}, \widetilde{\sigma}}^{\widehat{\alpha}} x_{\mu}^{\widetilde{\rho}} x_{\nu}^{\widetilde{\sigma}}+x_{\widetilde{\rho}}^{\widehat{\alpha}} x_{\mu, \nu}^{\widetilde{\rho}}
$$

By subtracting the corresponding expression for $x^{\widehat{\alpha}}{ }_{\nu, \mu}$, and multiplying by $x_{\widehat{\alpha}}^{\nu}$, we get

$$
x^{\nu}{ }_{\widehat{\alpha}}\left(x_{\mu, \nu}^{\widehat{\alpha}}-x_{\nu, \mu}^{\widehat{\alpha}}\right)=x_{\widehat{\alpha}}^{\widetilde{\sigma}_{\widehat{\alpha}}}\left(x_{\widetilde{\rho}, \widetilde{\sigma}}^{\widehat{\alpha}}-x_{\widetilde{\sigma}, \widetilde{\rho}}^{\widehat{\alpha}}\right) x_{\mu}^{\widetilde{\rho}}+x_{\widetilde{\rho}}^{\nu}\left(x_{\mu, \nu}^{\widetilde{\rho}}-x_{\nu, \mu}^{\widetilde{\rho}}\right),
$$

which is Eq. (9).

Validity of Equation (10). If we take the partial derivative of the equation $x^{\widetilde{\alpha}}{ }_{\mu}=\delta_{\mu}^{\alpha}+\delta_{0}^{\alpha} \delta_{\mu}^{2} x^{1}$ with respect to $x^{\nu}$, we get $x^{\widetilde{\alpha}}{ }_{\mu, \nu}=\delta_{0}^{\alpha} \delta_{\mu}^{2} \delta_{\nu}^{1}$. Subtraction of the corresponding expression with $\mu$ and $\nu$ interchanged gives

$$
x_{\mu, \nu}^{\widetilde{\alpha}}-x_{\nu, \mu}^{\widetilde{\alpha}}=\delta_{0}^{\alpha}\left(\delta_{\mu}^{2} \delta_{\nu}^{1}-\delta_{\nu}^{2} \delta_{\mu}^{1}\right) .
$$

If we choose $\widetilde{\alpha}=0, \quad \mu=2$, and $\nu=1$ we get

$$
x_{2,1}^{\widetilde{0}}-x_{1,2}^{\widetilde{0}}=\delta_{0}^{0}\left(\delta_{2}^{2} \delta_{1}^{1}-\delta_{1}^{2} \delta_{2}^{1}\right)=1,
$$

which shows that the transformation coefficients in Eq. (10) do not satisfy Eq. (11). However, upon multiplying Eq. (43) by $x_{\widetilde{\alpha}}^{\nu}=\delta_{\alpha}^{\nu}-\delta_{0}^{\nu} \delta_{\alpha}^{2} x^{1}$, we get

$$
x_{\widetilde{\alpha}}^{\nu}\left(x_{\mu, \nu}^{\widetilde{\alpha}}-x_{\nu, \mu}^{\widetilde{\alpha}}\right)=\delta_{\alpha}^{\nu} \delta_{0}^{\alpha}\left(\delta_{\mu}^{2} \delta_{\nu}^{1}-\delta_{\nu}^{2} \delta_{\mu}^{1}\right)-\delta_{0}^{\nu} \delta_{\alpha}^{2} x^{1} \delta_{0}^{\alpha}\left(\delta_{\mu}^{2} \delta_{\nu}^{1}-\delta_{\nu}^{2} \delta_{\mu}^{1}\right)=0
$$

which shows that the transformation coefficients in Eq. (10) do satisfy Eq. (4).

Equations (11) and (12). From $h_{\mu}^{i}=h_{\widetilde{\alpha}}^{i} x^{\widetilde{\alpha}}$ we see that

$$
h_{\mu, \nu}^{i}=h_{\widetilde{\alpha}, \nu}^{i} x_{\mu}^{\widetilde{\alpha}}+h_{\widetilde{\alpha}}^{i} x_{\mu, \nu}^{\widetilde{\alpha}}=h_{\widetilde{\alpha}, \widetilde{\beta}}^{i} x_{\mu}^{\widetilde{\alpha}} x_{\nu}^{\widetilde{\beta}}+h_{\widetilde{\alpha}}^{i} x_{\mu, \nu}^{\widetilde{\alpha}} .
$$

If we subtract the corresponding expression for $h_{\nu, \mu}^{i}$, and multiply by $h_{i}^{\nu}=$ $h_{i}^{\widetilde{\sigma}} x^{\nu} \widetilde{\sigma}$, we get

$$
h_{i}^{\nu}\left(h_{\mu, \nu}^{i}-h_{\nu, \mu}^{i}\right)=h_{i}^{\widetilde{\sigma}} x_{\widetilde{\sigma}}^{\nu}\left(h_{\widetilde{\alpha}, \widetilde{\beta}}^{i}-h_{\widetilde{\beta}}^{i}, \widetilde{\alpha}\right) x_{\mu}^{\widetilde{\alpha}} x^{\widetilde{\beta}}{ }_{\nu}+h_{i}^{\nu} h_{\widetilde{\alpha}}^{i}\left(x_{\mu, \nu}^{\widetilde{\alpha}}-x_{\nu, \mu}^{\widetilde{\alpha}}\right) .
$$

Thus, from the chain rule

$$
h_{i}^{\nu}\left(h_{\mu, \nu}^{i}-h_{\nu, \mu}^{i}\right)=h_{i}^{\widetilde{\beta}}\left(h_{\widetilde{\alpha}, \widetilde{\beta}}^{i}-h_{\widetilde{\beta}, \widetilde{\alpha}}^{i}\right) x_{\mu}^{\widetilde{\alpha}}+x_{\widetilde{\alpha}}^{\nu}\left(x_{\mu, \nu}^{\widetilde{\alpha}}-x_{\nu, \mu}^{\widetilde{\alpha}}\right) .
$$

This may be written

$$
C_{\mu}=C_{\widetilde{\alpha}} x^{\widetilde{\alpha}}+x_{\widetilde{\alpha}}^{\nu}\left(x_{\mu, \nu}^{\widetilde{\alpha}}-x_{\nu, \mu}^{\widetilde{\alpha}}\right)
$$

which is our Eq. (12). 
Derivation of Equation (15). The antisymmetry of $\gamma_{\mu \nu i}$ in $\mu$ and $\nu$ may be used to obtain an expression for $\gamma_{\mu \nu i}$ in terms of $f_{i \mu \nu}$. We have $f_{i \mu \nu}=$ $h_{i \nu, \mu}-h_{i \mu, \nu}=h_{i \nu ; \mu}-h_{i \mu ; \nu}$, so that

$$
f_{i \mu \nu}=\gamma_{i \nu \mu}-\gamma_{i \mu \nu}
$$

If we subtract the corresponding expressions for $f_{\mu \nu i}$ and $f_{\nu i \mu}$, we have

$$
\begin{aligned}
f_{i \mu \nu}-f_{\mu \nu i}-f_{\nu i \mu} & =\gamma_{i \nu \mu}-\gamma_{i \mu \nu}-\gamma_{\mu i \nu}+\gamma_{\mu \nu i}-\gamma_{\nu \mu i}+\gamma_{\nu i \mu} \\
= & -\gamma_{\nu i \mu}-\gamma_{i \mu \nu}+\gamma_{i \mu \nu}+\gamma_{\mu \nu i}+\gamma_{\mu \nu i}+\gamma_{\nu i \mu}=2 \gamma_{\mu \nu i}
\end{aligned}
$$

SO

$$
\gamma_{\mu \nu i}=\frac{1}{2}\left(f_{i \mu \nu}-f_{\mu \nu i}-f_{\nu i \mu}\right)
$$

which is our Eq. (15).

Equations (20) and (21). We note that $\sqrt{-g}$ equals $h$, the determinant of $h_{\mu}^{i}$, so we may write Eq. (20) as

$$
0=\int h\left(2 C^{j} C_{\nu}-C^{\alpha} C_{\alpha} h_{\nu}^{j}\right) \delta h_{j}^{\nu} \mathrm{d}^{4} x+\int 2 h C^{\mu} \delta C_{\mu} \mathrm{d}^{4} x
$$

We now need an expression for $\delta C_{\mu}$ to use in the last integral of Eq. (44). From $C_{\mu}=h_{j}{ }^{\nu}\left(h_{\mu, \nu}^{j}-h_{\nu, \mu}^{j}\right)$, we have

$$
\begin{aligned}
\delta C_{\mu} & =\left(h_{\mu, \nu}^{j}-h_{\nu, \mu}^{j}\right) \delta h_{j}^{\nu}+h_{j}{ }^{\nu}\left[\left(\delta h_{\mu}^{j}\right)_{{ }_{\nu}}-\left(\delta h_{\nu}^{j}\right)_{, \mu}\right] \\
& =\left(\gamma_{\mu \nu}^{j}-\gamma_{\nu \mu}^{j}\right) \delta h_{j}{ }^{\nu}+h_{j}{ }^{\nu}\left(\delta h_{\mu}^{j}\right)_{; \nu}-h_{j}{ }^{\nu}\left(\delta h_{\nu}^{j}\right)_{; \mu} .
\end{aligned}
$$

Upon multiplying by $2 h C^{\mu}$, we get an expression which we manipulate into the form

$$
\begin{aligned}
2 h C^{\mu} \delta C_{\mu}= & h\left(2 C^{\mu} \gamma^{j}{ }_{\mu \nu}-2 C^{\mu} \gamma^{j}{ }_{\nu \mu}\right) \delta h_{j}{ }^{\nu} \\
& +h\left(2 C_{; \alpha}^{\alpha} h_{i}{ }^{\beta}+2 C^{\mu} \gamma_{i}{ }^{\beta}{ }_{\mu}-2 C^{\beta}{ }_{; \mu} h_{i}{ }^{\mu}-2 C^{\beta} \gamma_{i}{ }_{\mu}{ }_{\mu}\right) \delta h_{\beta}{ }_{\beta} \\
& +\left(2 h C^{\mu} h_{j}{ }^{\nu} \delta h^{j}{ }_{\mu}-2 h C^{\nu} h_{j}{ }^{\mu} \delta h^{j}{ }_{\mu}\right)_{, \nu} \quad .
\end{aligned}
$$

We now need an expression for $\delta h_{\beta}^{i}$ in terms of $\delta h_{j}^{\nu}$. From $h_{\beta}^{j} h_{j}^{\nu}=\delta_{\beta}^{\nu}$ we get $h_{j}^{\nu} \delta h_{\beta}^{j}+h_{\beta}^{j} \delta h_{j}^{\nu}=0$, so $h_{\nu}^{i} h_{j}^{\nu} \delta h_{\beta}^{j}+h_{\nu}^{i} h_{\beta}^{j} \delta h_{j}^{\nu}=0$. Thus, we find that $\delta h_{\beta}^{i}=-h_{\nu}^{i} h_{\beta}^{j} \delta h_{j}^{\nu}$. Substituting this expression for $\delta h_{\beta}^{i}$ in the above 
expression for $2 h C^{\mu} \delta C_{\mu}$ gives

$$
\begin{aligned}
& 2 h C^{\mu} \delta C_{\mu}=h\left(2 C^{\mu} \gamma_{\mu \nu}^{j}-2 C^{\mu} \gamma_{\nu \mu}^{j}\right) \delta h_{j}{ }^{\nu} \\
& -h\left(2 C_{; \alpha}^{\alpha} h_{i}{ }^{\beta} h_{\nu}^{i} h_{\beta}^{j}+2 C^{\mu} \gamma_{i}{ }_{\mu}{ }_{\mu} h_{\nu}^{i} h_{\beta}^{j}\right) \delta h_{j}^{\nu} \\
& +h\left(2 C_{; \mu}^{\beta} h_{i}{ }^{\mu} h_{\nu}^{i} h_{\beta}^{j}+2 C^{\beta} \gamma_{i}{ }_{\mu}{ }_{\mu} h_{\nu}^{i} h_{\beta}{ }_{\beta}\right) \delta h_{j}{ }^{\nu} \\
& +\left(2 h C^{\mu} h_{j}{ }^{\nu} \delta h^{j}{ }_{\mu}-2 h C^{\nu} h_{j}{ }^{\mu} \delta h^{j}{ }_{\mu}\right){ }_{\nu} \\
& =h\left(2 C^{\mu} \gamma^{j}{ }_{\mu \nu}-2 C^{\mu} \gamma^{j}{ }_{\nu \mu}\right) \delta h_{j}{ }^{\nu} \\
& -h\left(2 C_{; \alpha}^{\alpha} h_{\nu}^{j}-2 C^{\mu} \gamma_{\nu \mu}^{j}\right) \delta h_{j}^{\nu} \\
& +h\left(2 C_{; \nu}^{\beta} h_{\beta}^{j}-2 C^{j} \gamma_{\nu \mu}^{\mu}\right) \delta h_{j}^{\nu} \\
& +\left(2 h C^{\mu} h_{j}{ }^{\nu} \delta h^{j}{ }_{\mu}-2 h C^{\nu} h_{j}{ }^{\mu} \delta h^{j}{ }_{\mu}\right),{ }_{\nu}
\end{aligned}
$$

We use $\gamma_{\nu \mu}^{\mu}=C_{\nu}$, and cancellations to obtain

$$
\begin{aligned}
2 h C^{\mu} \delta C_{\mu}= & h\left(2 C_{; \nu}^{\beta} h_{\beta}^{j}-2 C^{j} C_{\nu}+2 C^{\mu} \gamma_{\mu \nu}^{j}-2 C_{; \alpha}^{\alpha} h_{\nu}^{j}\right) \delta h_{j}{ }^{\nu} \\
& +\left(2 h C^{\mu} h_{j}{ }^{\nu} \delta h^{j}{ }_{\mu}-2 h C^{\nu} h_{j}{ }^{\mu} \delta h^{j}{ }_{\mu}\right)_{, \nu} .
\end{aligned}
$$

Thus, we have

$$
\begin{aligned}
\int 2 h C^{\mu} \delta C_{\mu} \mathrm{d}^{4} x= & \int h\left(2 C_{; \nu}^{\beta} h_{\beta}^{j}-2 C^{j} C_{\nu}+2 C^{\mu} \gamma_{\mu \nu}^{j}-2 C_{; \alpha}^{\alpha} h_{\nu}^{j}\right) \delta h_{j}{ }^{\nu} \mathrm{d}^{4} x \\
& +\int\left(2 h C^{\mu} h_{j}{ }^{\nu} \delta h^{j}{ }_{\mu}-2 h C^{\nu} h_{j}{ }^{\mu} \delta h_{\mu}^{j}{ }_{,{ }_{\nu}} \mathrm{d}^{4} x\right.
\end{aligned}
$$

and using this expression for $\int 2 h C^{\mu} \delta C_{\mu} d^{4} x$ in Eq. (44) gives

$$
\begin{aligned}
0 & =\int h\left(2 C_{; \nu}^{\beta} h_{\beta}^{j}+2 C^{\alpha} \gamma_{\alpha \nu}^{j}-2 C_{; \alpha}^{\alpha} h_{\nu}^{j}-C^{\alpha} C_{\alpha} h_{\nu}^{j}\right) \delta h_{j}{ }^{\nu} \mathrm{d}^{4} x \\
& +\int\left[2 h\left(C^{\mu} h_{j}{ }^{\nu}-C^{\nu} h_{j}{ }^{\mu}\right) \delta h^{j}{ }_{\mu}\right],_{\nu} \mathrm{d}^{4} x .
\end{aligned}
$$

By using Gauss's theorem, we may write the second integral above as an integral over the boundary of the region of integration. We discard this boundary integral by demanding that $\delta h^{j}{ }_{\mu}$ shall vanish on the boundary. Thus, we have

$$
0=\int h\left(2 C_{; \nu}^{\beta} h_{\beta}^{j}+2 C^{\alpha} \gamma_{\alpha \nu}^{j}-2 C_{; \alpha}^{\alpha} h_{\nu}^{j}-C^{\alpha} C_{\alpha} h_{\nu}^{j}\right) \delta h_{j}{ }^{\nu} \mathrm{d}^{4} x
$$

and by allowing the variation $\delta h_{j}{ }^{\nu}$ to be arbitrary in the interior of the region of integration, we obtain the Euler-Lagrange equations

$$
C_{; \nu}^{\beta} h_{\beta}^{j}+C^{\alpha} \gamma_{\alpha \nu}^{j}-C_{20}^{\alpha}{ }_{; \alpha} h_{\nu}^{j}-\frac{1}{2} C^{\alpha} C_{\alpha} h_{\nu}^{j}=0
$$


which are our field equations, but not in the form that we desire. If we multiply by $h_{j}^{\sigma} g_{\sigma \mu}$, we get

$$
C_{\mu ; \nu}-C_{\alpha} \gamma_{\mu \nu}^{\alpha}-g_{\mu \nu} C_{; \alpha}^{\alpha}-\frac{1}{2} g_{\mu \nu} C^{\alpha} C_{\alpha}=0 .
$$

This is our Eq. (21), i.e., our field equations as given in Sec. 5.1.

Noether Conserved Currents: The Antisymmetric Part of the Field Equations. We now apply Noether's theorem to the integral which was discarded after using Gauss's theorem. The integrand of this integral is

$$
2 h\left(C^{\mu} h_{j}{ }^{\nu}-C^{\nu} h_{j}{ }^{\mu}\right) \delta h^{j}{ }_{\mu} .
$$

A variation $\delta h^{j}{ }_{\mu}=g^{j n} \varepsilon_{n k} h^{k}$, where the $\varepsilon_{n k}$ are infinitesimal antisymmetric constants, leaves the metric unchanged. From this, and the straightforward use of Noether's theorem, we find that the six currents

$$
\mathfrak{J}_{i j}{ }^{\nu}=C_{\alpha}\left(h_{i}^{\alpha} h_{j}^{\nu}-h_{j}^{\alpha} h_{i}^{\nu}\right)
$$

satisfy the conservation laws $\mathfrak{J}_{i j: \nu}^{\nu}=0$. We note that $\mathfrak{J}_{i j}{ }^{\nu}$ is a vector under conservative coordinate transformations from $x^{\alpha}$ to $x^{\widetilde{\alpha}}$, and that these "proper" conservation laws may be expressed in the form $\left(\sqrt{-g} \mathfrak{J}_{i j}{ }^{\nu}\right)_{\nu}=0$. They are "weak" conservation laws in the sense that $\mathfrak{J}_{i j}{ }^{\nu}$ is conserved if the field equations are satisfied. Indeed, the antisymmetric part of Eq.(21) just states that $\mathfrak{J}_{i j: \nu}^{\nu}=0$.

Derivation of Eq. (23). Our identity for the Einstein tensor, Eq. (19), is

$$
\begin{aligned}
G_{\mu \nu}= & C_{\mu ; \nu}-C_{\alpha} \gamma_{\mu \nu}^{\alpha}-g_{\mu \nu} C_{; \alpha}^{\alpha}-\frac{1}{2} g_{\mu \nu} C^{\alpha} C_{\alpha} \\
& +\gamma_{\mu \nu ; \alpha}^{\alpha}+\gamma_{\sigma \nu}^{\alpha} \gamma_{\mu \alpha}^{\sigma}+\frac{1}{2} g_{\mu \nu} \gamma^{\alpha \sigma \beta} \gamma_{\beta \sigma \alpha},
\end{aligned}
$$

and we subtract the corresponding expression for $G_{\nu \mu}$ to obtain

$$
\begin{aligned}
G_{\mu \nu}-G_{\nu \mu}= & \left(C_{\mu ; \nu}-C_{\alpha} \gamma_{\mu \nu}^{\alpha}\right)-\left(C_{\nu ; \mu}-C_{\alpha} \gamma_{\nu \mu}^{\alpha}\right) \\
& +\left(\gamma_{\mu \nu ; \alpha}^{\alpha}+\gamma_{\sigma \nu}^{\alpha} \gamma_{\mu \alpha}^{\sigma}\right)-\left(\gamma_{\nu \mu ; \alpha}^{\alpha}+\gamma_{\sigma \mu}^{\alpha} \gamma_{\nu \alpha}^{\sigma}\right) .
\end{aligned}
$$

It is obvious that $G_{\mu \nu}-G_{\nu \mu}=R_{\mu \nu}-R_{\nu \mu}$, so we seek an identity for $R_{\mu \nu}-R_{\nu \mu}$ which is valid if the tetrad is path-dependent.

The covariant derivative of $h_{\beta}^{i}$ with respect to $x^{\mu}$ is given by

$$
h_{\beta ; \mu}^{i}=h_{\beta, \mu}^{i}-h_{\sigma}^{i} \Gamma_{\beta \mu}^{\sigma}
$$

where $\Gamma_{\mu \nu}^{\alpha}=\frac{1}{2} g^{\alpha \beta}\left(g_{\beta \mu, \nu}+g_{\beta \nu, \mu}-g_{\mu \nu, \beta}\right)$ is the usual Christoffel symbol. The covariant derivative of Eq. (46) with respect to $x^{\nu}$ gives

$$
\begin{aligned}
h_{\beta ; \mu ; \nu}^{i}= & h_{\beta, \mu, \nu}^{i}-h_{\sigma, \nu}^{i} \Gamma_{\beta \mu}^{\sigma}-h_{\sigma}^{i} \Gamma_{\beta \mu, \nu}^{\sigma}-h_{\sigma, \mu}^{i} \Gamma_{\beta \nu}^{\sigma} \\
& +h_{\sigma}^{i} \Gamma_{\rho \mu}^{\sigma} \Gamma_{\beta \nu}^{\rho}-h_{\beta, \sigma}^{i} \Gamma_{\mu \nu}^{\sigma}+h_{\sigma}^{i} \Gamma_{\beta \rho}^{\sigma} \Gamma_{\mu \nu}^{\rho} .
\end{aligned}
$$


If we subtract from Eq. (47) the corresponding expression with $\mu$ and $\nu$ interchanged, we get

$$
h_{\beta ; \mu ; \nu}^{i}-h_{\beta ; \nu ; \mu}^{i}=\left[\partial_{\nu}, \partial_{\mu}\right] h_{\beta}^{i}+h_{\sigma}^{i}\left(\Gamma_{\beta \nu, \mu}^{\sigma}-\Gamma_{\beta \mu, \nu}^{\sigma}+\Gamma_{\rho \mu}^{\sigma} \Gamma_{\beta \nu}^{\rho}-\Gamma_{\rho \nu}^{\sigma} \Gamma_{\beta \mu}^{\rho}\right),
$$

and if we multiply this by $h_{i}^{\alpha}$, we obtain

$$
R_{\beta \mu \nu}^{\alpha}=h_{i}^{\alpha}\left[\partial_{\nu}, \partial_{\mu}\right] h_{\beta}^{i}+\mathbb{R}_{\beta \mu \nu}^{\alpha}
$$

where

$$
\mathbb{R}_{\beta \mu \nu}^{\alpha}=\Gamma_{\beta \nu, \mu}^{\alpha}-\Gamma_{\beta \mu, \nu}^{\alpha}+\Gamma_{\rho \mu}^{\alpha} \Gamma_{\beta \nu}^{\rho}-\Gamma_{\rho \nu}^{\alpha} \Gamma_{\beta \mu}^{\rho}
$$

is the orthodox expression for the Riemann tensor in terms of the metric, rather than in terms of the tetrad. We see from Eq. (48) that

$$
R_{\mu \nu}=h_{i}^{\alpha}\left[\partial_{\nu}, \partial_{\alpha}\right] h_{\mu}^{i}+\mathbb{R}_{\mu \nu}
$$

where $\mathbb{R}_{\mu \nu}=\mathbb{R}_{\mu \alpha \nu}^{\alpha}$. Thus

$$
R_{\mu \nu}-R_{\nu \mu}=h_{i}^{\alpha}\left(\left[\partial_{\nu}, \partial_{\alpha}\right] h_{\mu}^{i}+\left[\partial_{\alpha}, \partial_{\mu}\right] h_{\nu}^{i}\right)+\mathbb{R}_{\mu \nu}-\mathbb{R}_{\nu \mu}
$$

We now compute the identity for $\mathbb{R}_{\mu \nu}-\mathbb{R}_{\nu \mu}$. We see from Eq. (49) that

$$
\mathbb{R}_{\alpha \beta \mu \nu}=g_{\alpha \sigma} \Gamma_{\beta \nu, \mu}^{\sigma}-g_{\alpha \sigma} \Gamma_{\beta \mu, \nu}^{\sigma}+[\alpha, \rho \mu] \Gamma_{\beta \nu}^{\rho}-[\alpha, \rho \nu] \Gamma_{\beta \mu}^{\rho}
$$

where $[\alpha, \mu \nu]=\frac{1}{2}\left(g_{\alpha \mu, \nu}+g_{\alpha \nu, \mu}-g_{\mu \nu}, \alpha\right)$. Thus, we have

$$
\begin{aligned}
\mathbb{R}_{\alpha \beta \mu \nu}= & \left(g_{\alpha \sigma} \Gamma_{\beta \nu}^{\sigma}\right)_{, \mu}-g_{\alpha \sigma, \mu} \Gamma_{\beta \nu}^{\sigma}-\left(g_{\alpha \sigma} \Gamma_{\beta \mu}^{\sigma}\right)_{, \nu}+g_{\alpha \sigma, \nu} \Gamma_{\beta \mu}^{\sigma} \\
& +[\alpha, \rho \mu] \Gamma_{\beta \nu}-[\alpha, \rho \nu] \Gamma_{\beta \mu}^{\rho}+[\alpha, \sigma \mu] \Gamma_{\beta \nu}^{\sigma}-[\alpha, \sigma \nu] \Gamma_{\beta \mu}^{\sigma} \\
= & {[\alpha, \beta \nu]_{, \mu}-[\alpha, \beta \mu]_{, \nu}+\left([\alpha, \sigma \mu]-g_{\alpha \sigma, \mu}\right) \Gamma_{\beta \nu}^{\sigma} } \\
& -\left([\alpha, \sigma \nu]-g_{\alpha \sigma, \nu}\right) \Gamma_{\beta \mu}^{\sigma} .
\end{aligned}
$$

Now,

$$
[\alpha, \sigma \mu]-g_{\alpha \sigma, \mu}=\frac{1}{2}\left(g_{\alpha \sigma, \mu}+g_{\alpha \mu, \sigma}-g_{\sigma \mu, \alpha}\right)-g_{\alpha \sigma, \mu}=-[\sigma, \alpha \mu] .
$$

From Eq. (153), we have $[\alpha, \sigma \mu]-g_{\alpha \sigma, \mu}=-[\sigma, \alpha \mu]$, and similarly $[\alpha, \sigma \nu]-g_{\alpha \sigma, \nu}=-[\sigma, \alpha \nu]$. Upon using these expressions in Eq. (52), we have

$$
\mathbb{R}_{\alpha \beta \mu \nu}=[\alpha, \beta \nu]_{, \mu}-[\alpha, \beta \mu]_{, \nu}-[\sigma, \alpha \mu] \Gamma_{\beta \nu}^{\sigma}+[\sigma, \alpha \nu] \Gamma_{\beta \mu}^{\sigma}
$$

so that

$$
\mathbb{R}_{\alpha \mu \beta \nu}=[\alpha, \mu \nu]_{, \beta}-[\alpha, \beta \mu]_{, \nu}-[\sigma, \alpha \beta] \Gamma_{\mu \nu}^{\sigma}+[\sigma, \alpha \nu] \Gamma_{\beta \mu}^{\sigma} .
$$

Upon multiplying Eq. (54) by $g^{\alpha \beta}$, we obtain

$$
\mathbb{R}_{\mu \nu}=g^{\alpha \beta}[\alpha, \mu \nu]_{, \beta}-g^{\alpha \beta}[\alpha, \beta \mu]_{, \nu}-g^{\alpha \beta}[\sigma, \alpha \beta] \Gamma_{\mu \nu}^{\sigma}+g^{\alpha \beta}[\sigma, \alpha \nu] \Gamma_{\beta \mu}^{\sigma}
$$

We see from Eq. (55) that

$$
\mathbb{R}_{\nu \mu}=g^{\alpha \beta}[\alpha, \nu \mu]_{, \beta}-g^{\alpha \beta}[\alpha, \beta \nu]_{, \mu}-g_{22}^{\alpha \beta}[\sigma, \alpha \beta] \Gamma_{\nu \mu}^{\sigma}+g^{\alpha \beta}[\sigma, \alpha \mu] \Gamma_{\beta \nu}^{\sigma} .
$$


We use the facts that $[\alpha, \nu \mu]=[\alpha, \mu \nu]$ and that $\Gamma_{\nu \mu}^{\sigma}=\Gamma_{\mu \nu}^{\sigma}$. Also, in the last term above, change $\alpha$ to $\beta$ and $\beta$ to $\alpha$. Thus, we obtain

$$
\mathbb{R}_{\nu \mu}=g^{\alpha \beta}[\alpha, \mu \nu]_{, \beta}-g^{\alpha \beta}[\alpha, \beta \nu]_{, \mu}-g^{\alpha \beta}[\sigma, \alpha \beta] \Gamma_{\mu \nu}^{\sigma}+g^{\alpha \beta}[\sigma, \beta \mu] \Gamma_{\alpha \nu}^{\sigma},
$$

Next, we use $\Gamma_{\alpha \nu}^{\sigma}=g^{\sigma \rho}[\rho, \alpha \nu]$ to obtain

$$
\mathbb{R}_{\nu \mu}=g^{\alpha \beta}[\alpha, \mu \nu]_{, \beta}-g^{\alpha \beta}[\alpha, \beta \nu]_{, \mu}-g^{\alpha \beta}[\sigma, \alpha \beta] \Gamma_{\mu \nu}^{\sigma}+g^{\alpha \beta}[\sigma, \alpha \nu] \Gamma_{\beta \mu}^{\sigma}
$$

Upon subtracting Eq. (56) from Eq. (55), we get

$$
\begin{aligned}
\mathbb{R}_{\mu \nu}-\mathbb{R}_{\nu \mu} & =g^{\alpha \beta}\left([\alpha, \beta \nu]_{, \mu}-[\alpha, \beta \mu]_{, \nu}\right) \\
& =\frac{1}{2} g^{\alpha \beta} g_{i j} h_{\beta}^{j}\left[\partial_{\mu}, \partial_{\nu}\right] h_{\alpha}^{i}+\frac{1}{2} g^{\alpha \beta} g_{i j} h_{\alpha}^{i}\left[\partial_{\mu}, \partial_{\nu}\right] h^{j}{ }_{\beta}
\end{aligned}
$$

Thus, with some obvious raising and lowering of indices, and a renaming of summed indices we obtain

$$
\mathbb{R}_{\mu \nu}-\mathbb{R}_{\nu \mu}=h_{i}^{\alpha}\left[\partial_{\mu}, \partial_{\nu}\right] h_{\alpha}^{i}
$$

We now use Eq. (57) in Eq. (51) to obtain

$$
R_{\mu \nu}-R_{\nu \mu}=G_{\mu \nu}-G_{\nu \mu}=h_{i}^{\alpha}\left(\left[\partial_{\nu}, \partial_{\alpha}\right] h_{\mu}^{i}+\left[\partial_{\alpha}, \partial_{\mu}\right] h_{\nu}^{i}+\left[\partial_{\mu}, \partial_{\nu}\right] h_{\alpha}^{i}\right)
$$

which is our desired identity, Eq. (23).

Consider a tetrad which satisfies the field equations and is path-independent in some $x^{\alpha}$ coordinate system. If this tetrad is transformed by a conservative transformation to an $x^{\widetilde{\alpha}}$ coordinate system, the transformed tetrad also satisfies the field equations, but is generally path-dependent. Thus, any statement that a tetrad is, or is not, path-independent has no invariant meaning. As stated in Sec. 5.2, we suspect that any tetrad which satisfies the field equations admits a conservative transformation to a coordinate system in which it is path-independent, but we have not succeeded in proving this.

Equations (24) and (30). The analysis for manipulating the right-side of Eq. (24) into a form such that its physical significance is transparent is without approximation, but is rather tedious and not entirely straightforward. Therefore, we now give this analysis in some detail:

We see from Eq. (26) and Eq. (27) that the totally antisymmetric part and the mixed symmetry part of $\gamma_{\mu \alpha \nu}$ are $A_{\mu \alpha \nu}=\frac{1}{3}\left(\gamma_{\mu \alpha \nu}+\gamma_{\alpha \nu \mu}+\gamma_{\nu \mu \alpha}\right)$ and $M_{\mu \alpha \nu}=\frac{1}{3}\left(2 \gamma_{\mu \alpha \nu}-\gamma_{\alpha \nu \mu}-\gamma_{\nu \mu \alpha}\right)$, respectively, and we recall that $M$ is antisymmetric in its first two indices. Thus, we have $\gamma_{\mu \nu}^{\alpha}=A_{\mu \nu}^{\alpha}+M_{\mu \nu}^{\alpha}$ and $\gamma_{\nu \mu}^{\alpha}=A_{\nu \mu}^{\alpha}+M_{\nu \mu}^{\alpha}$. Since $A_{\mu \nu}^{\alpha}+A_{\nu \mu}^{\alpha}=0$, we see that $\gamma_{\mu \nu}^{\alpha}+\gamma_{\nu \mu}^{\alpha}=$ $M_{\mu \nu}^{\alpha}+M_{\nu \mu}^{\alpha}$. By using this in Eq. (24), we obtain

$$
T_{\mu \nu}=\frac{1}{2}\left(M_{\mu \nu ; \alpha}^{\alpha}+M_{\nu \mu ; \alpha}^{\alpha}+\gamma_{\sigma \nu}^{\alpha} \gamma_{\mu \alpha}^{\sigma}+\gamma_{\sigma \mu}^{\alpha} \gamma_{\nu \alpha}^{\sigma}+g_{\mu \nu} \gamma^{\alpha \sigma \beta} \gamma_{\beta \sigma \alpha}\right) \text {. }
$$


Now, $M_{\mu \nu ; \alpha}^{\alpha}=\left(M_{\mu i}^{\alpha} h_{\nu}^{i}\right)_{; \alpha}=M_{\mu i ; \alpha}^{\alpha} h_{\nu}^{i}+M_{\mu i}^{\alpha} h_{\nu ; \alpha}^{i}=M_{\mu i ; \alpha}^{\alpha} h_{\nu}^{i}+M_{\mu \sigma}^{\alpha} \gamma_{\nu \alpha}^{\sigma}$, so we have

$$
M_{\mu \nu ; \alpha}^{\alpha}=J_{\mu i} h_{\nu}^{i}+M_{\mu \sigma}^{\alpha} \gamma_{\nu \alpha}^{\sigma}
$$

where $J_{\mu i}=M_{\mu i ; \alpha}^{\alpha}$ is the current which is the source of the field $M_{\mu \nu i}$. Upon using Eq. (60) and the corresponding expression for $M_{\nu \mu ; \alpha}^{\alpha}$ in Eq. (59) we get

$$
\begin{aligned}
T_{\mu \nu}= & \frac{1}{2}\left(J_{\mu i} h_{\nu}^{i}+J_{\nu i} h_{\mu}^{i}+M_{\mu \sigma}^{\alpha} \gamma_{\nu \alpha}^{\sigma}+M_{\nu \sigma}^{\alpha} \gamma_{\mu \alpha}^{\sigma}\right) \\
& +\frac{1}{2}\left(\gamma_{\sigma \nu}^{\alpha} \gamma_{\mu \alpha}^{\sigma}+\gamma_{\sigma \mu}^{\alpha} \gamma_{\nu \alpha}^{\sigma}+g_{\mu \nu} \gamma^{\alpha \sigma \beta} \gamma_{\beta \sigma \alpha}\right)
\end{aligned}
$$

We now express each Ricci rotation coefficient in Eq. (61) as the sum of its antisymmetric and its mixed-symmetry parts. Thus, we have

$$
\begin{aligned}
T_{\mu \nu}= & \frac{1}{2}\left[J_{\mu i} h_{\nu}^{i}+J_{\nu i} h_{\mu}^{i}+M_{\mu \sigma}^{\alpha}\left(A_{\nu \alpha}^{\sigma}+M_{\nu \alpha}^{\sigma}\right)+M_{\nu \sigma}^{\alpha}\left(A_{\mu \alpha}^{\sigma}+M_{\mu \alpha}^{\sigma}\right)\right] \\
& +\frac{1}{2}\left[\left(A_{\sigma \nu}^{\alpha}+M_{\sigma \nu}^{\alpha}\right)\left(A_{\mu \alpha}^{\sigma}+M_{\mu \alpha}^{\sigma}\right)+\left(A_{\sigma \mu}^{\alpha}+M_{\sigma \mu}^{\alpha}\right)\left(A_{\nu \alpha}^{\sigma}+M_{\nu \alpha}^{\sigma}\right)\right] \\
& +\frac{1}{2}\left[g_{\mu \nu}\left(A^{\alpha \sigma \beta}+M^{\alpha \sigma \beta}\right)\left(A_{\beta \sigma \alpha}+M_{\beta \sigma \alpha}\right)\right] .
\end{aligned}
$$

Upon clearing the inner parentheses, changing the order of terms, and using the total antisymmetry of $A$ and antisymmetry of $M$ in its first two indices, we obtain after a little factoring

$$
\begin{aligned}
T_{\mu \nu}= & \frac{1}{2}\left[J_{\mu i} h_{\nu}^{i}+J_{\nu i} h_{\mu}^{i}+M_{\mu \sigma}^{\alpha}\left(M_{\nu \alpha}^{\sigma}+M_{\alpha \nu}^{\sigma}\right)+M_{\nu \sigma}^{\alpha}\left(M_{\mu \alpha}^{\sigma}+M_{\alpha \mu}^{\sigma}\right)\right] \\
& +\frac{1}{2}\left[A_{\mu \alpha}{ }^{\sigma}\left(M_{\nu \sigma}^{\alpha}+M_{\sigma \nu}^{\alpha}+M_{\sigma \nu}^{\alpha}\right)+A_{\nu \alpha}^{\sigma}\left(M_{\mu \sigma}^{\alpha}+M_{\sigma \mu}^{\alpha}+M_{\sigma \mu}^{\alpha}\right)\right] \\
& +A_{\mu \sigma}^{\alpha} A_{\alpha \nu}{ }^{\sigma}+\frac{1}{2} g_{\mu \nu}\left(2 A^{\alpha \sigma \beta} M_{\beta \sigma \alpha}+M^{\alpha \sigma \beta} M_{\beta \sigma \alpha}-A^{\alpha \beta \sigma} A_{\alpha \beta \sigma}\right)
\end{aligned}
$$

It follows from Eq. (27) that

$$
M_{\mu \nu i}+M_{i \mu \nu}+M_{\nu i \mu}=0 .
$$

We easily find from Eqs. (26) and (27) that

$$
M^{\mu \nu \alpha} A_{\mu \nu \alpha}=0 \text {. }
$$

Now

$$
M^{\alpha \sigma \beta} M_{\beta \sigma \alpha}=\frac{1}{2} M^{\alpha \sigma \beta} M_{\beta \sigma \alpha}+\frac{1}{2} M^{\alpha \sigma \beta} M_{\beta \sigma \alpha}=\frac{1}{2} M^{\alpha \sigma \beta} M_{\beta \sigma \alpha}+\frac{1}{2} M^{\sigma \alpha \beta} M_{\beta \alpha \sigma}
$$

and since $\mathbf{M}$ is antisymmetric in its first two indices,

$$
M^{\alpha \sigma \beta} M_{\beta \sigma \alpha}=\frac{1}{2} M^{\alpha \sigma \beta} M_{\beta \sigma \alpha}+\frac{1}{2} M^{\alpha \sigma \beta} M_{\alpha \beta \sigma}=\frac{1}{2} M^{\alpha \sigma \beta}\left(M_{\beta \sigma \alpha}+M_{\alpha \beta \sigma}\right) .
$$

Obviously Eq. (62) implies that $M_{\beta \sigma \alpha}+M_{\alpha \beta \sigma}=-M_{\sigma \alpha \beta}=M_{\alpha \sigma \beta}$ so we see that

$$
M^{\alpha \sigma \beta} M_{\beta \sigma \alpha}=\frac{1}{2} M^{\alpha \sigma \beta} M_{\alpha \sigma \beta} .
$$


Thus, we have

$$
\begin{aligned}
& T_{\mu \nu}=\frac{1}{2}\left(J_{\mu i} h_{\nu}^{i}+J_{\nu i} h_{\mu}^{i}-M_{\mu \sigma}^{\alpha} M_{\nu \alpha}^{\sigma}-M_{\nu \sigma}^{\alpha} M_{\mu \alpha}^{\sigma}\right)+A_{\mu \sigma}^{\alpha} A_{\alpha \nu}^{\sigma} \\
& +\frac{1}{2} g_{\mu \nu}\left(2 A^{\alpha \sigma \beta} M_{\beta \sigma \alpha}+M^{\alpha \sigma \beta} M_{\beta \sigma \alpha}-A^{\alpha \beta \sigma} A_{\alpha \beta \sigma}\right) .
\end{aligned}
$$

Therefore, Eq. (65) reduces to

$$
\begin{aligned}
T_{\mu \nu}= & \frac{1}{2}\left(J_{\mu i} h_{\nu}^{i}+J_{\nu i} h_{\mu}^{i}\right)-\left(M_{\mu \alpha}{ }^{\sigma} M_{\nu \sigma}^{\alpha}-\frac{1}{4} g_{\mu \nu} M^{\alpha \sigma \beta} M_{\alpha \sigma \beta}\right) \\
& +A^{\alpha}{ }_{\mu \sigma} A_{\alpha \nu}{ }^{\sigma}-\frac{1}{2} g_{\mu \nu} A^{\alpha \beta \sigma} A_{\alpha \beta \sigma} .
\end{aligned}
$$

If we use the total antisymmetry of $\mathbf{A}$, the antisymmetry of $\mathbf{M}$ in its first two indices, raise and lower some summed indices, and change some Greek indices to Latin indices, we obtain

$$
\begin{aligned}
T_{\mu \nu}= & \frac{1}{2}\left(J_{\mu i} h_{\nu}^{i}+J_{\nu i} h_{\mu}^{i}\right)-\left(M_{\mu i}^{\alpha} M_{\alpha \nu}{ }^{i}-\frac{1}{4} g_{\mu \nu} M^{\alpha \sigma i} M_{\alpha \sigma i}\right) \\
& +A^{i j}{ }_{\mu} A_{i j \nu}-\frac{1}{2} g_{\mu \nu} A^{i j \alpha} A_{i j \alpha} .
\end{aligned}
$$

The terms in Eq. (67) that involve A may be written in a more simple form. We define a vector

$$
A^{\mu}=\frac{1}{3 !}(-g)^{-1 / 2} e^{\mu \alpha \beta \sigma} A_{\alpha \beta \sigma},
$$

where $e^{\mu \alpha \beta \sigma}$ is the usual Levi-Civita tensor density of weight +1 . Thus,

$$
\begin{aligned}
A_{\nu} & =A^{\mu} g_{\mu \nu}=\frac{1}{3 !}(-g)^{-1 / 2} e^{\mu \alpha \beta \sigma} g_{\mu \nu} A_{\alpha \beta \sigma}=\frac{1}{3 !}(-g)^{-1 / 2} e^{\mu \alpha \beta \sigma} g_{\mu \nu} g_{\alpha \rho} g_{\beta \gamma} g_{\sigma \tau} A^{\rho \gamma \tau} \\
& =\frac{1}{3 !}(-g)^{-1 / 2} g e_{\nu \rho \gamma \tau} A^{\rho \gamma \tau}=-\frac{1}{3 !}(-g)^{1 / 2} e_{\nu \rho \gamma \tau} A^{\rho \gamma \tau} .
\end{aligned}
$$

We see from the above that we have the equivalent expressions

$$
A_{\nu}=-\frac{1}{3 !}(-g)^{1 / 2} e_{\nu \rho \gamma \tau} A^{\rho \gamma \tau}
$$

and

$$
A_{\nu}=\frac{1}{3 !}(-g)^{-1 / 2} e^{\mu \alpha \beta \sigma} g_{\mu \nu} A_{\alpha \beta \sigma} .
$$

From the second expression for $A_{\nu}$, we have

$$
A_{\mu}=\frac{1}{3 !}(-g)^{-1 / 2} e^{\kappa \alpha \beta \sigma} g_{\kappa \mu} A_{\alpha \beta \sigma} .
$$

By multiplying Eqs. (68) and (69), we get

$$
A_{\mu} A_{\nu}=-\frac{1}{36} e^{\kappa \alpha \beta \sigma} e_{\nu \rho \gamma \tau} g_{\kappa \mu} A_{\alpha \beta \sigma} A^{\rho \gamma \tau} .
$$

We now express the product of Levi-Civita symbols as a determinant of Kronecker deltas (see. e.g. Weber [40]), and find that

$$
A_{\mu} A_{\nu}=\frac{1}{2} A^{i j}{ }_{\mu} A_{i j \nu}-\frac{1}{6} g_{\mu \nu} A^{i j \alpha} A_{i j \alpha}
$$

and if we multiply by $g^{\mu \nu}$ we easily find that

$$
A^{i j \alpha} A_{i j \alpha}=-6 A^{\alpha} A_{\alpha} .
$$


We find from the last two equations that

$$
A_{\mu}^{i j} A_{i j \nu}=2 A_{\mu} A_{\nu}-2 g_{\mu \nu} A^{\alpha} A_{\alpha} .
$$

Now, by substituting the above expressions for $A^{i j \alpha} A_{i j \alpha}$ and $A^{i j}{ }_{\mu} A_{i j \nu}$ into Eq. (67), we obtain the more simple expression, which is our Eq. (30) in Sec. 5.3.

$$
\begin{aligned}
T_{\mu \nu}= & \frac{1}{2}\left(J_{\mu i} h_{\nu}^{i}+J_{\nu i} h_{\mu}^{i}\right)-\left(M_{\mu i}^{\alpha} M_{\alpha \nu}{ }^{i}-\frac{1}{4} g_{\mu \nu} M^{\alpha \sigma i} M_{\alpha \sigma i}\right) \\
& +2 A_{\mu} A_{\nu}+g_{\mu \nu} A^{\alpha} A_{\alpha} .
\end{aligned}
$$

Equations (36) and (37). We have

$$
\begin{aligned}
\gamma^{\mu i \nu} \gamma_{\mu \nu i} & =\left(A^{\mu i \nu}+M^{\mu i \nu}\right)\left(A_{\mu \nu i}+M_{\mu \nu i}\right) \\
& =A^{\mu i \nu} A_{\mu \nu i}+A^{\mu i \nu} M_{\mu \nu i}+M^{\mu i \nu} A_{\mu \nu i}+M^{\mu i \nu} M_{\mu \nu i}
\end{aligned}
$$

and, from Eq. (63), we see that $A^{\mu i \nu} M_{\mu \nu i}=M^{\mu i \nu} A_{\mu \nu i}=0$. Thus,

$$
\gamma^{\mu i \nu} \gamma_{\mu \nu i}=-A^{\mu \nu i} A_{\mu \nu i}+M^{i \mu \nu} M_{\nu \mu i}
$$

From Eq. (70) we have $-A^{\mu \nu i} A_{\mu \nu i}=6 A^{\alpha} A_{\alpha}$, and from Eq. (64) we have $M^{i \mu \nu} M_{\nu \mu i}=\frac{1}{2} M^{\mu \nu i} M_{\mu \nu i}$. Hence

$$
\gamma^{\mu i \nu} \gamma_{\mu \nu i}=\frac{1}{2} M^{\mu \nu i} M_{\mu \nu i}+6 A^{\alpha} A_{\alpha}
$$

as claimed above Eq. (37).

\section{REFERENCES}

1. Einstein, A.: Autobiographical notes. in Albert Einstein: Philosopher-

Scientist, edited by P. A. Schilpp, Harper \& Brothers, New York, Vol. I, p. 89 (1949)

2. Dirac, P. A. M.: The Principles of Quantum Mechanics, Cambridge University Press, Preface to First Edition (1930)

3. Pandres, D., Jr.: On forces and interactions between fields. J. Math. Phys. 3, 602-607 (1962)

4. Pandres, D., Jr.: Unification of gravitation and electromagnetism. Lett.

Nuovo Cimento 8, 595-599 (1973)

5. Pandres, D., Jr.: Gravitation and electromagnetism. Found. Phys. 7, 421-430 (1977)

6. Feynman, R. P., Hibbs, A. R.: Quantum Mechanics and Path Integrals, McGraw Hill (1965)

7. Witten, E.: in Superstrings: A Theory of Everything, edited by P. C. W. Davis, J. Brown: Cambridge University Press, p. 90 (1988)

8. Eddington, A. S.: The Mathematical Theory of Relativity, 2nd Ed., Cambridge University Press, p. 225 (1924) 
9. Penrose, R.: Int. J. Theor. Phys. 1, 61 (1968)

10. Penrose, R., MacCallum, A. H.: Phys. Rep. 6C, 242 (1972) and

references contained therein

11. Finkelstein, D.: Phys. Rev. 184, 1261 (1969)

12. Finkelstein, D.: Phys. Rev. D 5, 321

13. Finkelstein, D., Frye, G., Susskind, L.: Phys. Rev. D 9, 2231 (1974) and references contained therein

14. Bergmann, P. G., Komar, A.: The coordinate group symmetries of general relativity, Int. J. Theor. Phys. 5, 15-28 (1972)

15. Gambini, R., Trias, A.: Geometrical origin of gauge theories, Phys. Rev. D15 23, 553-555 (1981)

16. Connes, A. "Noncommutative differential geometry and the structure of space time," Prepared for NATO Advance Study Institute on Quantum Fields and Quantum Space Time, Cargese, France (1996)

17. Crane, L. "What Is the mathematical structure of quantum space-time?", Los Alamos Preprints (LANL Number 0706.4452v1), June 29, 2007.

18. Mandelstam, S.: Quantum electrodynamics without potentials, Ann.

Phys., N.Y. 19, 1-24 (1962)

19. Pandres, D., Jr.: Quantum unified field theory from enlarged coordinate transformation group. Phys. Rev. D, 24, 1499-1508 (1981)

20. Pauli, W.: Theory of Relativity, Pergamon Press, Oxford, p. 5 (1958)

21. Pandres, D., Jr.: Quantum unified field theory from enlarged coordinate transformation group II. Phys. Rev. D, 30, 317-324 (1984)

22. Synge, J. L.: Relativity: The General Theory, North-Holland Publishing Company, Amsterdam, p. 117, 357 (1960)

23. Eisenhart, L. P.: Riemannian Geometry, Princeton University Press (1964)

24. Taub, A. H.: in Perspectives in Geometry and Relativity, edited by Hoffmann, B., Indiana University Press, Bloomington, p. 360 (1966)

25. Schrödinger, E.: Space-Time Structure, Cambridge University Press, p. 97,99 (1960)

26. Pandres, D., Jr.: Unified gravitational and Yang-Mills fields. Int. J. Theor. Phys. 34, 733-759 (1995)

27. Pandres, D., Jr.: Gravitational and electroweak unification. Int. J.

Theor. Phys. 38, 1783-1805 (1999)

28. Salam, A: in Proceedings of the 8th Nobel Symposium on Elementary

Particle Theory, N. Svartholm, ed., Almquist Forlag, Stockholm, 367 (1967)

29. Weinberg, S.: A model of leptons, Phys. Rev. Lett. 19, 1264 (1967)

30. Yang, C.N., Mills, R.L.: Conservation of isotopic spin and isotopic gauge invariance, Phys. Rev. 96, 191 (1954) 
31. Nakahara, M.: Geometry, Topology and Physics, Adam Hilger, New York, p. 344 (1990)

32. Moriyasu, K.: An Elementary Primer for Gauge Theory, World Scientific, Singapore, p. 52 (1983)

33. Pandres, D., Jr, Green, E. L.: Unified field theory from enlarged transformation group: The inconsistent Hamiltonian. Int. J. Theor. Phys. 42, 1849-1873 (2003)

34. Bergmann, P. G.,Goldberg, I.: Dirac bracket transformations in phase space, Phys. Rev. 98, 531-538 (1955)

35. Dirac, P. A M.: Lectures on Quantum Mechanics, Academic Press, New York (1964)

36. Sundermeyer, K.: Constrained Dynamics, Springer-Verlag, Berlin (1982)

37. Weinberg S.: The Quantum Theory of Fields, Vol. I, Cambridge University Press, (1995)

38. Pandres, D., Jr.: Quantum geometry from coordinate transformations relating quantum observers. Int. J. Theor. Phys. 23, 839-842 (1984)

39. Everett, H. III: Relative state formulation of quantum mechanics, Revs. Mod. Phys. 29, 454-462 (1957)

40. Weber, J.: General Relativity and Gravitational Waves, Interscience Publishers, New York, p. 147 (1961) 